\title{
IMPACTOS RECIENTES DE LOS CAMBIOS AMBIENTALES EN LOS RECURSOS HÍDRICOS SUPERFICIALES DE LA CUENCA DEL DUERO
}

\author{
Impact of environmental change un surface \\ water resources in the Douro basin
}

\author{
ENRIQUE MORÁN TEJEDA \\ Instituto Pirenaicos de Ecología, CSIC (Spanish Research Council), Campus de Aula Dei, \\ P.O. Box 202, Zaragoza 50080, Spain.enrique.moran@unige.ch
}

\begin{abstract}
In recent decades, the environmental changes due to human development have put pressure on water resources in the Mediterranean basin, a region where availability of water has been historically limited. In this work we analyze the evolution and variability (1961-2005) of streamflows in one of the largest rivers basin of the Iberian Peninsula, the Duero River basin. Moreover, the factors responsible for such evolution are assessed. Results show a significant and generalized decrease of water resources in the basin, together with changes in the timing of monthly distribution. Climate itself, with quasi-stationary precipitation and increasing temperatures in the long-term, is not enough to explain the decreasing streamflows. Thus, observed land-cover expansion in the headwaters is thought to be increasingly contributing to the hydrological depletion. On the other hand, impoundment of water through dams is increasing in the basin and consequently contributing the hydrological change. Results offer the basis for future projections of water availability in scenarios of water scarcity due to forthcoming climate change
\end{abstract}

Keywords: Duero basin, water resources, fluvial regime, climate variability, land-cover, hydrological management.

RESUMEN.- La disponibilidad de recursos hídricos ha sido históricamente un factor limitante de desarrollo en los países de la cuenca mediterránea. Durante las últimas décadas la presión sobre la disponibilidad se ha acentuado debido a los cambios ambientales observados como resultado del desarrollo industrial. En este trabajo se analizan la evolución y variabilidad recientes (1961-2005) de los recursos hidri- 
cos superficiales - el caudal en los ríos-en una de las cuencas hidrográficas de mayor entidad de la Península Ibérica, y los factores ambientales responsables de su evolución. Los resultados del trabajo muestran un descenso notable y generalizado en los caudales en la región, acompañado de un cambio en los regímenes fluviales. La evolución del clima, con unas precipitaciones muy variables pero sin tendencias notables a largo plazo, y unas temperaturas en aumento, explica en parte, pero no en su totalidad, el descenso hidrológico. En las cabeceras fluviales se ha detectado un incremento significativo de la cubierta vegetal durante el periodo de estudio, el cual parece estar participando en gran medida en el descenso de caudales. Por otro lado, la regulación por medio de embalses está incrementando en la cuenca y con ello contribuyendo al cambio hidrológico en la región. Los resultados obtenidos ofrecen la base conceptual para proyectar la disponibilidad futura de los recursos hídricos en los escenarios de mayor escasez como consecuencia del cambio climático venidero.

Palabras clave: Cuenca del Duero, recursos hídricos, régimen fluvial, variabilidad climática, cubierta vegetal, gestión hidrológica.

\section{Introducción}

La cuenca del río Duero es por su extensión y por el volumen de agua drenada, uno de los sistemas hidrográficos de mayor entidad de la Península Ibérica. Si bien su régimen hidrológico no tiene el carácter deficitario de las cuencas de la vertiente mediterránea, la presión a la que están sometidos los recursos hídricos, tanto por factores naturales como antrópicos, hace de su evaluación una tarea fundamental si se quiere pronosticar la disponibilidad futura los mismos. La localización de la cuenca en el noroeste peninsular y su disposición topográfica a modo de gran meseta rodeada de cadenas montañosas le confieren unos rasgos climáticos diversos, destacando la mediterraneidad y la continentalidad del interior y los ambientes húmedos y fríos del reborde montañoso y el sector noroccidental más cercano al océano Atlántico. Las montañas que bordean la cuenca son, gracias a su capacidad de retención y almacenamiento de las precipitaciones, la fuente principal de los recursos hídricos de la región, siendo ésta una característica común de las montañas en los ambientes mediterráneos (Viviroli \& Weingartner 2004, García-Ruiz et al., 2011). Los recursos hídricos de la cuenca, su cantidad y variabilidad, dependerán por lo tanto en gran medida de los procesos que tengan lugar en las zonas de montaña. Los gradientes altitudinales y energéticos confieren además a las montañas una especial sensibilidad frente a los cambios ambientales (Beniston, 2005), por lo que el estudio de los mismos es fundamental para comprender la disponibilidad de los recursos hídricos aguas abajo, donde se produce mayoritariamente su consumo. 
Durante las dos últimas décadas han proliferado en la literatura científica los estudios enfocados a evaluar la evolución reciente de los recursos hídricos y los procesos relacionados con la misma (p.ej. Lettenmaier et al., 1994, Kahya \& Kalayci, 2004, Birsan et al., 2005, García-Ruiz et al., 2011). El motivo principal es la preocupación existente sobre la disponibilidad de agua en las décadas futuras, en las que se pronostican cambios graduales en los valores de las principales variables climáticas (temperaturas y precipitaciones) en el contexto del denominado "cambio climático". De forma muy general un calentamiento de la atmósfera a nivel planetario, derivado del aumento en la concentración de gases invernadero, incrementará los ratios de evapotranspiración provocando a su vez un incremento en la variabilidad espacial y temporal de las precipitaciones y causando notables cambios en el ciclo hidrológico (Milly et al., 2005, IPCC, 2007).

No obstante, en los últimos años también se ha puesto el énfasis en el papel hidrológico de un proceso que se observa de forma generalizada en las zonas de montaña de los países desarrollados: el incremento de la cubierta vegetal, como consecuencia del abandono de las actividades tradicionales de pastoreo y cultivo en las laderas de montaña. Los trabajos realizados en cuencas experimentales han demostrado que la cubierta vegetal y los bosques afectan al ciclo hidrológico y el balance de agua en la medida en que controlan los procesos de partición de la precipitación (Crockford \& Richardson, 2000, Llorens \& Domingo, 2007), antes de formar parte de la escorrentía superficial: la cubierta vegetal, o el dosel arbóreo retiene parte de las gotas de lluvia en el proceso denominado como "interceptación", y consume agua para sus necesidades vitales, potenciando ambos procesos la evapotranspiración del agua a la atmósfera; asimismo favorece la infiltración de agua en el suelo a través de sus sistemas radiculares(Zhang et al., 2001, Cosandey et al., 2005, Morán, 2007).Por ello cualquier cambio de uso que suponga un incremento en densidad o superficie de la misma repercute directamente en un descenso en la escorrentía, mientras que una retirada de la cubierta vegetal suele aumentar los caudales de los ríos (p. ej., Bosch \& Hewlett, 1982, Bent, 2001, Gallart \& Llorens, 2003).

Desde mediados del siglo XX se viene advirtiendo en la montaña española un incremento paulatino de la cubierta vegetal. Los dos factores que más peso han tenido en esta recuperación han sido el abandono de las actividades agrarias en las zonas de montaña, y las repoblaciones forestales. El abandono de la agricultura o la disminución de la presión del pastoreo sobre el suelo montano provoca una regeneración natural de los ecosistemas de montaña mediante la recolonización vegetal, que puede evolucionar hasta etapas más avanzadas, como el establecimiento de una cubierta forestal (Vicente-Serrano et al,. 2000). Este proceso ha sido observado en diferentes sistemas montaño- 
sos españoles como los Pirineos (Poyatos et al., 2003) o el Sistema Ibérico (Lasanta-Martínez et al., 2005). Junto a ello, la sustitución de cultivos cerealistas por pastizales y las reforestaciones llevadas a cabo por las administraciones han sido los procesos de cambio más significativos que ha experimentado el suelo de la montaña española en las últimas décadas (García-Ruiz et al., 1996).

Así como la variabilidad climática es un condicionante natural de la disponibilidad de los recursos hídricos, y el aumento de la cubierta vegetal responde a procesos mixtos de intervención antrópica y evolución natural de los ecosistemas, los caudales de los ríos de la mayor parte de sistemas hidrográficos mundiales están sujetos a fuertes regulaciones estacionales e hiperanuales a través de las presas. La construcción de embalses constituye uno de los impactos más destacados del hombre sobre el medio natural (Petts, 1984). Los embalses y su gestión causan alteraciones en el régimen de los ríos, la disminución de caudales aguas abajo como resultado de la infiltración y evaporación de la lámina de agua, alteran los ritmos y tasas de erosión/sedimentación de los ríos al actuar como trampas de sedimentos, y modifican incluso las propiedades físico-químicas del agua, por lo que inducen a graves alteraciones en los ecosistemas riparios (Cosandey\& Robinson, 2000, Verstraeten \& Poesen, 2000, Maingi \& Marsh, 2002, Bonacci \& Roje-Bonacci, 2003, Nilsson et al., 2005). Pero quizás el impacto más estremecedor lo produzcan sobre las poblaciones humanas, con la desaparición de poblaciones enteras, desplazamientos masivos de personas, pérdida de campos de cultivo en las superficies inundadas, incluso pérdidas de vidas humanas producidas por accidentes (Berkun, 2010). No obstante, y a pesar de sus impactos negativos, numerosos embalses han sido construidos y se siguen construyendo en todo el mundo para atender a diferentes demandas. Entre sus funciones más importantes destacan el aprovisionamiento de agua para la irrigación de cultivos, la producción hidroeléctrica, el control y laminación de avenidas o el suministro urbano e industrial (López-Moreno et al., 2002). En la cuenca del Duero existen a día de hoy más de 90 embalses, con una capacidad cercana a los 7.500 $\mathrm{hm}^{3}$, y una demanda total de $3.870 \mathrm{hm}^{3}$. De dicho volumen más del $93 \%$, $\left(3.600 \mathrm{hm}^{3}\right)$ se destina al regadío, aproximadamente el 6\% $\left(225 \mathrm{hm}^{3}\right)$ se destina a abastecimientos urbanos y domésticos, y el resto, unos $45 \mathrm{hm}^{3}$ a usos industriales y otros (www.chduero.es). Además, los embalses con una localización topográfica propicia para los saltos de agua son utilizados también para la producción de energía hidroeléctrica.

El presente trabajo comprende una valoración global de la evolución de los recursos hídricos superficiales en la cuenca del Duero, de su variabilidad espacial y temporal durante la segunda mitad del siglo pasado y primeros años del presente, teniendo en cuenta los tres factores mencionados líneas 
arriba. Se analizan por lo tanto los patrones de cambio en las variables climáticas (temperaturas y precipitaciones), usos del suelo y gestión de embalses y en qué medida han afectado a la evolución de los caudales de los ríos de la cuenca durante el periodo 1961-2005. Los resultados obtenidos servirán como punto de partida para contextualizar trabajos enfocados a inferir la disponibilidad futura de los recursos hídricos a través de la modelización hidrológica.

\section{Zona de estudio: la cuenca del Duero}

La totalidad de la cuenca del Duero cubre una extensión de 97.290 km², si bien este trabajo se ha enfocado en el sector español de la cuenca, que ocupa un $81 \%$ de dicha superficie y drena a Portugal unos aportes anuales de 13.800 $\mathrm{hm}^{3}$ a través del su río principal, el Duero (Figura 1). La mayor parte de la cuenca del Duero se corresponde con una depresión colmatada con sedimentos terciarios y cuaternarios, conformando una meseta de una altitud media relativamente elevada $(700 \mathrm{~m})$, y en la que se ha asentado la red fluvial desde la era terciaria. Desde el punto de vista hidrológico las unidades más importantes son los bordes montañosos ya que constituyen el área fuente de los recursos hídricos. Los bordes de la cuenca corresponden a diferentes cadenas montañosas que alcanzan altitudes superiores a $\operatorname{los} 2.500 \mathrm{~m}$, y tienen un origen geológico y composición litológica heterogénea: i) Los Montes de León o Montañas Galaico-Leonesas representan el límite noroccidental de la cuenca, están formadas por materiales paleozoicos y presentan altitudes superiores a los $2.000 \mathrm{~m}$. ii) El límite norte lo forma la Cordillera Cantábrica, con cumbres próximas a los $2.500 \mathrm{~m}$. y una estructura geológica compleja en cuanto a su origen, con materiales diversos del Paleozoico y Cenozoico. iii) El Sistema Ibérico delimita la parte oriental de la cuenca y está formado por materiales mesozoicos con cimas que superan los 2.000 m. iv) Finalmente, por el sur la cuenca está delimitada por el Sistema Central con materiales paleozoicos -plutónicos y metamórficos-, fracturados y deformados por la orogenia alpina, y cumbres que alcanzan los $2.500 \mathrm{~m}$.

El régimen térmico de la cuenca es el característico de un clima de interior, con una apreciable oscilación térmica anual, entre las temperaturas inferiores a los $5^{\circ} \mathrm{C}$ en invierno, y los $20^{\circ} \mathrm{C}$ de media en verano. Por su parte, el régimen pluviométrico es la característica que define la mediterraneidad del clima, con un período húmedo entre el otoño y la primavera y un período con escasa pluviosidad en los meses estivales. Espacialmente las precipitaciones se distribuyen de forma desigual entre el interior (se sobrepasan los $400 \mathrm{~mm}$ anuales) y el borde montañoso, donde se registran con más de $1.000 \mathrm{~mm}$ anuales. A pesar del predominio de un clima mediterráneo continentalizado 
en la mayor parte de la cuenca, en los rebordes montañosos sería más apropiado hablar de climas tanto sub-mediterráneos como sub-atlánticos.

Por último, la dualidad existente en la topografía de la cuenca también está presente en la distribución de los usos del suelo y de las formaciones vegetales. Con la excepción de pequeñas manchas forestales o bosques de ribera, el interior de la cuenca está enteramente ocupado por terrenos de cultivo dedicados al cereal, el viñedo y el regadío. El dominio forestal se extiende por el sector suroccidental con formaciones de encina (Quercus ilexsp.ballota) y melojos (Q. pirenaica), y en las cadenas montañosas, con un marcado contraste bioclimático que explica la presencia de abedulares (Betula pendula), hayedos (Fagus sylvatica) y robledales (Q. robur) en las montañas septentrionales, y de melojares y pinares ( $P$. pinaster y $P$. sylvestris) en las montañas orientales y meridionales.

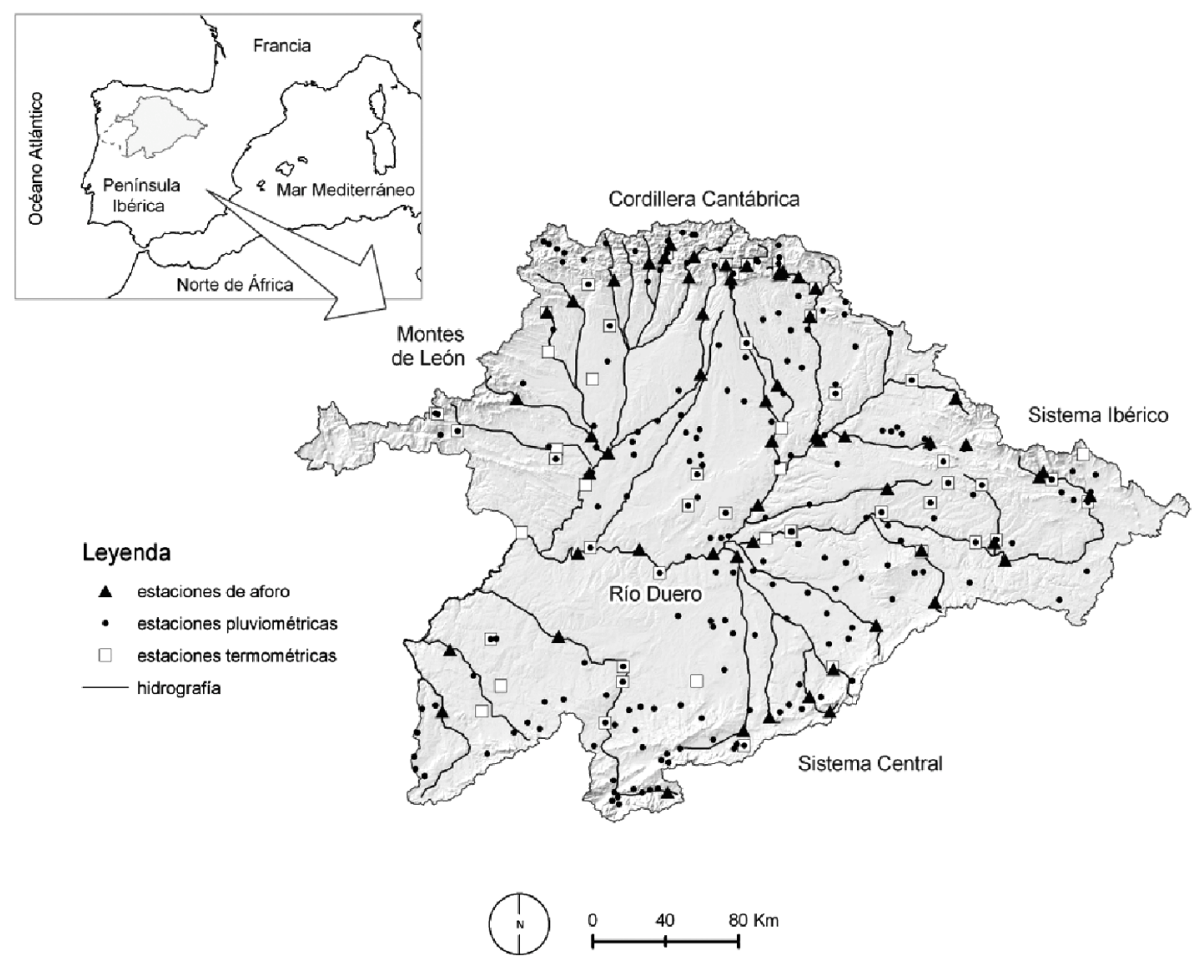

Figura 1. La cuenca del Duero y la localización de las estaciones hidrológicas y climáticas estudiadas.

Figure 1. The Duero basin and the location of streamflows and weather stations used. 
IMPACTOS RECIENTES DE LOS CAMBIOS AMBIENTALES EN LOS RECURSOS HÍDRICOS...

\section{Datos y métodos}

En esta sección presentamos un resumen de los métodos y técnicas utilizados para alcanzar los objetivos generales del trabajo. Se obvia la exposición de los procedimientos estadísticos habituales sin perjuicio de que aparezcan mencionados a lo largo del texto y, para más detalles, se remite al lector interesado a las referencias bibliográficas citadas.

\subsection{Datos climáticos, hidrológicos y cartográficos}

Una base de datos climáticos, hidrológicos y de usos del suelo ha sido elaborada para todo el territorio de la cuenca. Los datos climáticos, precipitaciones $(\mathrm{mm})$ y temperaturas medias mensuales $\left({ }^{\circ} \mathrm{C}\right)$, para el periodo 1961-2005 fueron obtenidos de la Agencia Española de Meteorología (AEMET). Los datos hidrológicos, aportaciones fluviales $\left(\mathrm{hm}^{3}\right)$ mensuales y caudales diarios $\left(\mathrm{m}^{3} / \mathrm{s}\right)$, de la Confederación Hidrográfica del Duero, y los usos del suelo de los mapas forestales de España de 1966 a escala 1:400.000 en papel, y del 2003 a escala 1:50.000 y en formato digital, para las 9 provincias castellano-leonesas.

Tanto los datos climáticos como hidrológicos pasaron un control de calidad basado en la detección de inhomogeneidades, en el relleno de datos ausentes, y en la eliminación de datos erróneos u outliers (ver detalles de dichos procedimientos en: Alexandersson, 1986, Lanzante, 1996, Peterson et al., 1998, García-Ruiz et al., 2001, Vicente-Serrano et al., 2009). Tras el proceso, que resultó en el descarte de un número considerable de series al no cumplir los requisitos de calidad (periodo de registro 1961-2005 y porcentaje de datos ausentes $<15 \%$ ), un total de 214 series de precipitación, 57 series de temperatura y 56 series de aportaciones fluviales, con una distribución más o menos homogénea en la cuenca (Figura 1), fueron utilizadas para los análisis.

Para analizar las variaciones en los usos del suelo se cartografiaron los mismos sobre los mapas forestales originales citados líneas arriba. Estos mapas contienen la cartografía de tipos de cubierta vegetal pero también de usos del suelo, como por ejemplo las formaciones forestales (pinos, esclerófilas, y decíduas), matorrales, pastizales, campos de cultivo y cuerpos de agua. Sin embargo, el número y tipología de clases difieren enormemente entre las dos fuentes (1966 y 2003). Por ello se realizó un reclasificación y homogeneización de leyendas mediante la fusión de las clases originales en función de una similar cubierta del suelo. El resultado fueron cinco simples clases de uso del suelo: agua, urbano-suelo desnudo, cultivo, pastizal-matorral y bosque. Esto permitió el cálculo de la variación de superficie ocupada por cada clase entre las dos fechas señaladas. 
ENRIQUE MORÁN TEJEDA

\subsection{Análisis de tendencias}

Además de la aplicación de pruebas estadísticas estándares como los análisis de correlación y regresión lineal, el análisis central del trabajo se basa en la detección de tendencias en las series de datos, cuya metodología detallamos continuación:

Se entiende como tendencia al cambio gradual a largo plazo en la media de una variable. Un procedimiento común para ilustrar si una serie de datos presenta una tendencia es el ajuste de una recta (regresión lineal) a la serie temporal, lo que permite comprobar la dirección (positiva o negativa) y magnitud (grado de inclinación) de dicha tendencia. Sin embargo, ésta se trata de una prueba paramétrica, esto es, que se basa en los valores de las series, y es por lo tanto vulnerable a la existencia de datos extremos, o a la no-normalidad en la distribución de los datos. Para solventar este problema, desde hace décadas se han desarrollado las denominadas pruebas "no-paramétricas", que se basan en el rango de los registros dentro de la serie en vez de en sus valores. Los test no-paramétricos más utilizados en la detección de tendencias han sido, y son, el test de rangos de Spearman(Lehmann, 1975, Sneyers, 1990) y el test de Mann-Kendall (Mann, 1945, Kendall, 1975). La validez y robustez de ambos en la detección de tendencias en series climáticas e hidrológicas ha sido ampliamente demostrada, siendo muy similar los resultados que de ellos se obtienen (Hirsch et al., 1982, Berryman et al., 1988, Zhang et al., 2000, Yue et al., 2002). El test de Mann-Kendall ha sido utilizado para la detección de tendencias en las series hidrológicas y climáticas de este trabajo,después de comprobar la similitud de resultados con el test de Spearman. La hipótesis nula $\left(\mathrm{N}_{0}\right)$ representa el caso en que no existe un cambio gradual en la media de la serie de datos a lo largo del tiempo; y la hipótesis alternativa $\left(\mathrm{N}_{1}\right)$ correspondería con el caso en el cual la media está aumentando o disminuyendo a lo largo del tiempo (Kundzewicz \& Robson 2004). El test se ha aplicado mediante el paquete estadístico SPSS ${ }^{\circledR}$, y se basa en un algoritmo de correlación entre la variable $X$ (años) y la variable $Y$ (precipitación, temperaturas, y aportaciones hídricas mensuales de cada año). Las observaciones se ordenan, separadamente, de forma ascendente y son reemplazadas por sus rangos. El test de Mann-Kendall se basa en el estadístico S, que se define como:

$$
S=\sum_{i=1}^{n-1} \sum_{j=i+1}^{n} \operatorname{sgn}\left(x_{j}-x_{i}\right)
$$


dondeson los valores de la secuencia de datos, es la longitud de la serie de datos y

$$
\operatorname{sgn}(\phi)=\left\{\begin{array}{ccc}
1 & \text { si } & \phi>0 \\
0 & \text { si } & \phi=0 \\
-1 & \text { si } & \phi<0
\end{array}\right\}
$$

El test de Mann-Kendall nos devuelve dos parámetros que nos permiten identificar la existencia de tendencias en las series así como su magnitud. El primero de ellos es el estadístico tau $\tau$, y nos indica el signo (positivo o negativo) de la tendencia, y su intensidad. Un tau $=0$ indica la inexistencia de tendencia. Cuando los valores se alejan de cero, estaríamos ante la existencia de una tendencia, positiva si los valores son positivos y negativa si los valores son negativos. El segundo parámetro, y quizás el más importante, es la significación estadística $(\alpha)$, que mide, en términos de probabilidad, si el valor de la pendiente obtenida es diferente del rango de valores que tendría bajo la hipótesis nula. Ello nos indica, por lo tanto, la probabilidad de que podamos rechazar o no, con certeza, la hipótesis nula.

\subsection{Análisis de componentes principales}

En un estudio de carácter regional como el presente, uno de los asuntos más interesantes es la identificación de patrones o modelos de comportamiento común en distintos parámetros de las variables estudiadas, y comprobar si éstos presentan una distribución espacial determinada. Gracias al avance de las ciencias de la información y a la existencia de bases de datos cada vez más extensas, se ha hecho cada vez más frecuente la búsqueda de técnicas que permiten identificar patrones, comúnmente denominadas "herramientas de reducción de datos". En el presente trabajo se ha utilizado en distintas ocasiones el conocido como "Análisis de Componentes Principales", cuyo potencial en la detección de patrones climáticos e hidrológicos es ampliamente reconocido (p.ej. Widmann \& Schär, 1997, Rodríguez-Puebla et al., 1998, Kalayci \& Kahya, 2006).

El análisis de componentes principales (PCA) es una técnica de análisis factorial, esto es, que se basa en la extracción de factores que definen la estructura subyacente de un universo multivariante (matriz de datos). El objetivo general de esta técnica es reducir la dimensionalidad de la base datos formada por un número elevado de variables, y obtener grupos de variables, independientes entre sí y que retienen la mayor parte de la varianza contenida en las variables originales (Tabachnick \& Fidell, 1996). Para que el análisis sea 
efectivo, las variables originales tienen que mostrar entre sí altos valores de correlación, lo cual significa que existe información redundante y que por ello puede ser reducida y explicada por una serie de variables nuevas. Las nuevas variables obtenidas (componentes principales) son combinaciones lineales de las variables originales y no muestran correlación entre sí (Jollife, 2002). El primer componente principal es aquella combinación lineal que explica el mayor porcentaje de varianza contenida en las variables originales, el segundo componente será la combinación lineal no correlacionada con el componente anterior y que absorbe el mayor porcentaje de varianza no explicada por el primero, y así sucesivamente.

\section{Resultados}

\subsection{Evolución y tendencias climáticas}

En la Figura 2 se muestran los mapas anual y mensuales de tendencias, resultado de interpolar (mediante un método kriging) los valores del tau de Mann-Kendall en cada serie de temperaturas anuales y mensuales. La figura refleja que en la totalidad de la cuenca las temperaturas medias anuales han experimentado una tendencia creciente durante el periodo de estudio. En todo el territorio el incremento térmico ha sido estadísticamente significativo (con un 95\% de confianza) y asimismo parece existir una tendencia hacia un aumento más acentuado en la zona sureste de la cuenca. En cuanto a las temperaturas medias mensuales, en todos los meses se han registrado tendencias positivas en la mayor parte del territorio de la cuenca, aunque no con la misma intensidad ni significación estadística. Los meses en los que las tendencias han sido más acentuadas son marzo, junio, agosto, y en menor medida mayo y diciembre. Por su parte, enero, febrero, julio, septiembre y octubre y noviembre son los que ha registrado, aunque positivas, tendencias más suaves, y no significativas en la mayor parte del territorio. Estacionalmente se puede decir que han sido en los meses de primavera y verano donde el ascenso térmico ha sido más pronunciado; y en el otoño donde el incremento ha sido más atenuado. 
IMPACTOS RECIENTES DE LOS CAMBIOS AMBIENTALES EN LOS RECURSOS HÍDRICOS...

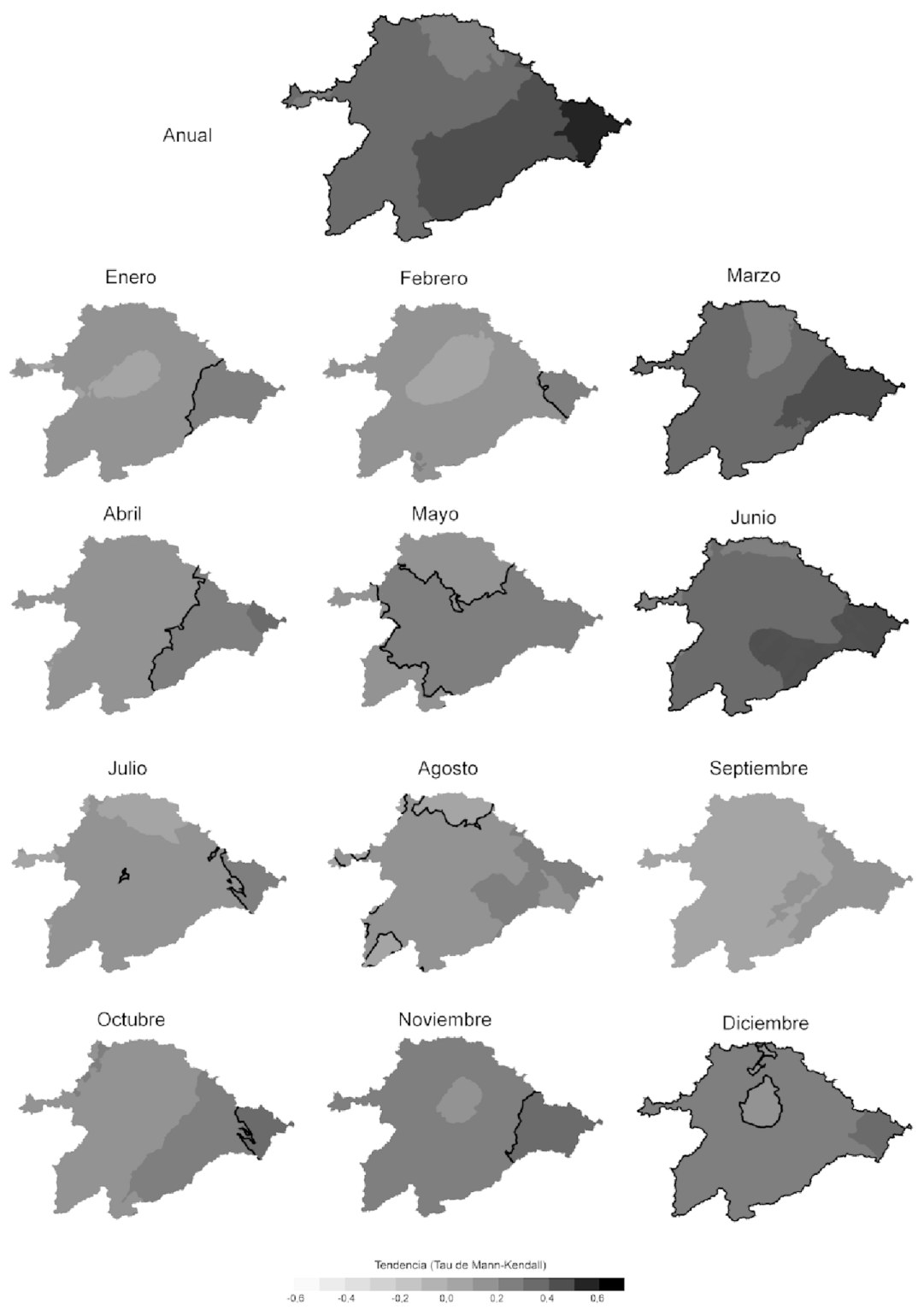

Figura 2. Tendencias de las temperaturas medias anuales y mensuales en la cuenca del Duero durante el periodo de estudio. Línea negra: $\alpha<0,05$ (estadísticamente significativo con un 95\% de confianza).

Figure 2. Trends in mean annual and monthly temperatures in the Duero basin, for the studied period. Black line: $\alpha<0,05$ (significant with a $95 \%$ of confidence). 
El test de Mann-Kendall aplicado a una serie promedio para toda la región (Tabla 1) muestra una tendencia creciente acentuada y estadísticamente significativa con un $99 \%$ de confianza; además un ajuste lineal realizado entre las temperaturas y el tiempo revela un cambio entre el comienzo y el final del periodo superior a un $11 \%$, pasando de una media de $12,2^{\circ} \mathrm{C}$ de temperatura predicha en 1961 a $13,6^{\circ} \mathrm{C}$ en el 2005 . Esto supone una variación de $+1,4^{\circ} \mathrm{C}$ a lo largo del periodo de estudio, o lo que es lo mismo, un aumento de casi $0,3^{\circ} \mathrm{C}$ por década. El desglose mensual del ajuste lineal revela un aumento de en torno $2^{\circ} \mathrm{C}$ en el mes de marzo y agosto, y de $2,3^{\circ} \mathrm{C}$ en junio, es decir de casi $0,4^{\circ} \mathrm{C}$ y $0,5^{\circ} \mathrm{C}$ por década respectivamente. Septiembre y octubre son por su parte los meses en los que el incremento ha sido menor, con apenas $0,5^{\circ} \mathrm{C}$ de diferencia entre el comienzo y el final del periodo.

Tabla 1. Estadísticos de centralidad y cambio (ajuste lineal y test de Mann-Kendall) para las series regionales de temperaturas mensuales y anuales. * Indica estadísticamente significativo con un $95 \%$ de confianza.

Table 1. Basic statistics for the regional series of temperature. *Indicates significant with a 95\% of confidence.

\begin{tabular}{|c|c|c|c|c|c|c|c|}
\hline \multirow[b]{2}{*}{$n=53$} & \multirow{2}{*}{$\begin{array}{l}\text { Tempera- } \\
\text { tura } \\
\text { media }\left({ }^{\circ} \mathrm{C}\right)\end{array}$} & \multicolumn{4}{|c|}{ Ajuste lineal } & \multicolumn{2}{|c|}{ Tendencia } \\
\hline & & $R^{2}$ & $\begin{array}{c}\text { Predicho } \\
1961\end{array}$ & $\begin{array}{c}\text { Predicho } \\
2005\end{array}$ & Cambio \% & $\operatorname{tau} M K$ & $\alpha$ \\
\hline Enero & 1,96 & 0,07 & 1,50 & 2,34 & 56,00 & $0,21^{*}$ & 0,04 \\
\hline Febrero & 2,77 & 0,07 & 2,29 & 3,19 & 39,30 & 0,16 & 0,13 \\
\hline Marzo & 4,35 & $0,33^{*}$ & 3,34 & 5,38 & 61,08 & $0,41^{*}$ & 0,00 \\
\hline Abril & 9,57 & $0,09^{*}$ & 5,00 & 5,92 & 18,40 & $0,21^{*}$ & 0,04 \\
\hline Mayo & 13,60 & $0,10^{*}$ & 7,40 & 8,52 & 15,14 & $0,23^{*}$ & 0,03 \\
\hline Junio & 17,73 & $0,38^{*}$ & 9,60 & 11,90 & 23,96 & $0,46^{*}$ & 0,00 \\
\hline Julio & 20,11 & $0,21^{*}$ & 12,20 & 13,50 & 10,66 & $0,29 *$ & 0,01 \\
\hline Agosto & 12,69 & $0,34^{*}$ & 11,70 & 13,60 & 16,24 & $0,41^{*}$ & 0,00 \\
\hline Septiembre & 10,55 & 0,03 & 10,15 & 10,80 & 6,40 & 0,11 & 0,29 \\
\hline Octubre & 7,43 & 0,03 & 7,10 & 7,70 & 8,45 & 0,12 & 0,25 \\
\hline Noviembre & 4,18 & $0,08^{*}$ & 3,70 & 4,60 & 24,32 & 0,17 & 0,10 \\
\hline Diciembre & 2,41 & $0,17^{*}$ & 1,70 & 3,10 & 82,35 & $0,30^{*}$ & 0,00 \\
\hline AÑO & 12,90 & $0,21^{*}$ & 12,16 & 13,55 & 11,43 & $0,29^{*}$ & 0,01 \\
\hline
\end{tabular}

En la Figura 3 se muestran los mapas de tendencias en las precipitaciones anuales y mensuales. En ellos se observa que no existe una homogeneidad en el signo de las tendencias en los distintos meses del año, además de que, en la mayoría de los casos, los valores de tendencia no son significativos, o sólo 
IMPACTOS RECIENTES DE LOS CAMBIOS AMBIENTALES EN LOS RECURSOS HÍDRICOS...

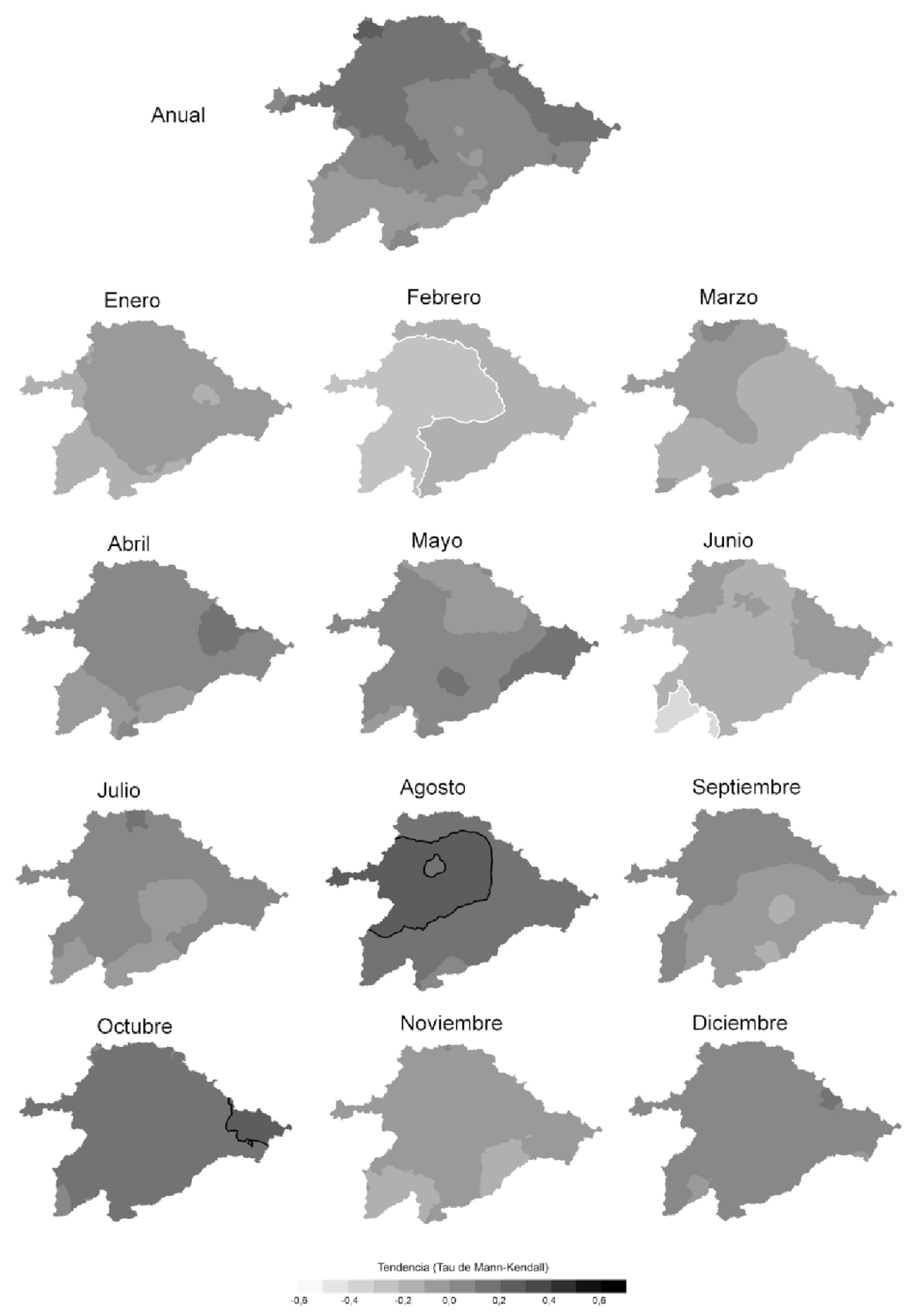

Figura 3. Tendencias de las precipitaciones anuales y mensuales en la cuenca del Duero durante el periodo de estudio. Línea negra (tendencias positivas) y blanca (tendencia negativa): $\alpha<0,05$ (estadísticamente significativo con un $95 \%$ de confianza).

Figure 3. Trends in annual and monthly precipitation in the Duero basin for the studied period. Black (positive trend) and white (negative trend) lines: $\alpha<0,05$ (significant with a 95\% of confidence). 
lo son en pequeñas partes del territorio. Los meses de octubre, diciembre, abril, mayo, julio y agosto presentan tendencias positivas, pero tan solo en agosto éstas son significativas en una parte importante del territorio. En términos de ganancia o pérdida de agua de precipitación las tendencias más importantes son aquellas registradas para los meses de invierno, ya que estos meses acumulan la mayor parte de la precipitación. En cualquier caso, estas tendencias negativas (tampoco las positivas) no son estadísticamente significativas y generalizadas en todo el territorio de la cuenca, y ello se ve reflejado asimismo en las tendencias para las precipitaciones anuales. A pesar de que se aprecia un patrón espacial en la distribución de las tendencias, con valores positivos en la mitad norte de la cuenca, y valores negativos en la mitad sur, tan sólo en una pequeña zona del sector suroccidental las tendencias son estadísticamente significativas, mientras que en la mayor parte del territorio los valores de tendencia no son significativos con un $95 \%$ de confianza.

La Tabla 2 muestra además los estadísticos de dispersión y cambio (incluyendo las tendencias) para las precipitaciones medias en la cuenca; también se muestran las tendencias (tau de Mann-Kendall) y su significación estadística. En la mayoría de los meses no se registran tendencias significativas, excepto en junio, con una tendencia regresiva, y en agosto, con una tendencia positiva. Las precipitaciones de febrero y octubre también han registrado una tendencia negativa y positiva, respectivamente, con unos valores que se encuentran en el límite de la significación estadística establecida. Mediante un ajuste lineal se ha estimado el porcentaje de cambio que se ha producido entre el comienzo y el final del periodo. Para los meses citados anteriormente, las precipitaciones han ascendido en más de un $130 \%$ y $104 \%$ en agosto y octubre, respectivamente, y se han reducido en un $56 \%$ y un $24 \%$ en febrero y junio. No obstante, la importancia de tales cambios, independientemente de su magnitud, radica en el volumen de precipitaciones registrado en cada mes. Por ello los más importantes a considerar son los meses de febrero y octubre (con tendencias opuestas), cuyas precipitaciones representan cerca de un 20\% del total anual. Por el contrario, un aumento del $130 \%$ en las precipitaciones de agosto, no supondrá una gran contribución a la evolución de las precipitaciones anuales, dado su escaso peso relativo (3\%). Como resultado, las precipitaciones anuales muestran una evolución, dentro de su variabilidad, más o menos estacionaria en el tiempo; permitiendo el test de Mann-Kendall confirmar la inexistencia de una tendencia significativa para el periodo de estudio $(\operatorname{tau}=0,04 ; \alpha=0,72)$. 
IMPACTOS RECIENTES DE LOS CAMBIOS AMBIENTALES EN LOS RECURSOS HÍDRICOS...

Tabla 2. Estadísticos de centralidad, dispersión, y cambio para las precipitaciones medias de la cuenca del Duero durante el periodo 1961-2005. * Estadísticamente significativo con un 95\% de confianza.

Table 2. Basic statistics for the regional series of precipitation. *Indicates significant with a 95\% of confidence.

\begin{tabular}{|c|c|c|c|c|c|c|c|}
\hline \multirow{2}{*}{$n=214$} & \multirow{2}{*}{$\begin{array}{c}\text { Precipi- } \\
\text { tación } \\
\text { media } \\
(\mathrm{mm})\end{array}$} & \multirow{2}{*}{$\begin{array}{l}\text { Contri- } \\
\text { bución } \\
\text { al total } \\
\text { anual \% }\end{array}$} & \multirow{2}{*}{\begin{tabular}{|c|} 
Coeficiente \\
de varia- \\
ción \\
medio $\%$
\end{tabular}} & \multicolumn{2}{|c|}{ Ajuste lineal } & \multicolumn{2}{|c|}{ Tendencia } \\
\hline & & & & $R^{2}$ & Cambio \% & $\tan M K$ & - \\
\hline Enero & 67,73 & 10,68 & 79,33 & 0,01 & $-13,89$ & $-0,09$ & 0,38 \\
\hline Febrero & 55,98 & 8,83 & 84,48 & 0,11 & $-56,41$ & $-0,18$ & 0,08 \\
\hline Marzo & 46,95 & 7,40 & 80,85 & 0,02 & $-24,53$ & $-0,10$ & 0,35 \\
\hline Abril & 57,85 & 9,12 & 65,47 & 0,00 & 8,93 & $-0,01$ & 0,92 \\
\hline Mayo & 62,86 & 9,91 & 59,48 & 0,02 & 18,97 & 0,08 & 0,45 \\
\hline Junio & 39,63 & 6,25 & 79,17 & 0,08 & $-24,00$ & $-0,23^{*}$ & 0,02 \\
\hline Julio & 21,67 & 3,42 & 112,43 & 0,00 & 4,55 & 0,01 & 0,89 \\
\hline Agosto & 19,81 & 3,12 & 108,27 & 0,09 & 133,33 & $0,24^{*}$ & 0,02 \\
\hline Septiembre & 40,55 & 6,39 & 83,86 & 0,00 & $-8,33$ & $-0,01$ & 0,95 \\
\hline Octubre & 68,31 & 10,77 & 75,58 & 0,11 & 104,44 & 0,20 & 0,05 \\
\hline Noviembre & 74,16 & 11,69 & 73,86 & 0,00 & $-7,79$ & $-0,07$ & 0,48 \\
\hline Diciembre & 70,97 & 11,19 & 82,07 & 0,02 & 43,10 & 0,07 & 0,51 \\
\hline Año & 634,29 & 100,00 & 23,33 & 0,00 & 0,16 & 0,04 & 0,72 \\
\hline
\end{tabular}

\subsection{Cambios en los usos del suelo}

La Figura 4 muestra el estado de los usos del suelo según los mapas forestales de 1966 y 2003 y la reclasificación efectuada (detalles en el apartado metodológico). A pesar de la diferencia de detalle (debido a las distintas escalas de los mapas originales), es fácil apreciar visualmente el crecimiento que se ha producido en la superficie cubierta por el bosque entre 1966 y 2003. De acuerdo con los análisis espaciales (Tabla 3), el crecimiento de la superficie forestal ha sido de un $14,2 \%$, pasando a ocupar poco más de $20.000 \mathrm{~km}^{2}$ en 1966, a casi 23.500 en el 2003. La superficie correspondiente al pastizal-matorral también ha crecido en la misma proporción que el bosque, con un $15 \%$ de variación. La expansión de ambos se ha producido en detrimento del suelo ocupado por cultivos y uso agrícola, que, siendo el principal uso del suelo de la cuenca, ha sufrido un retroceso del 16\% entre las dos fechas. Si consideramos el análisis comparado de las franjas norte y sur de la cuenca, donde se sitúan las cabeceras de la mayoría de los ríos que drenan al Duero, observamos que existen notables diferencias en la variación de los usos entre ambos territorios. Así, la superficie forestal ha aumentado en más de un 30\%, mientras que en la fran- 
ja sur se ha producido un incremento más moderado de la superficie forestal, con un $18 \%$ de variación.

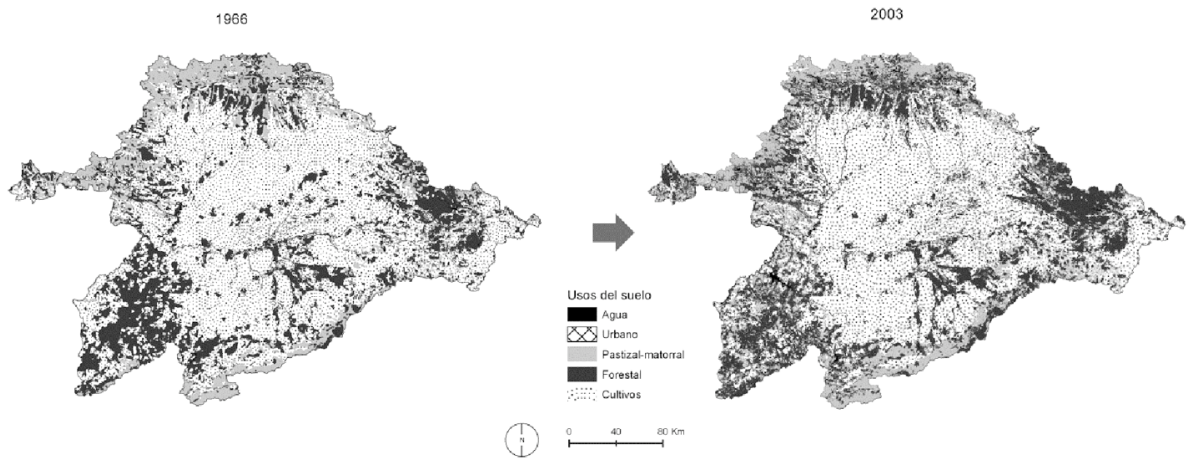

Figura 4. Variación de los usos del suelo en la cuenca del Duero entre 1966 y 2003. Figure 4. Land-use changes in the Duero basin between 1966 and 2003.

Tabla 3. Variación de la superficie $\left(\mathrm{km}^{2}\right)$ ocupada por los distintos usos del suelo Table 3. Variation of the surface (square kilometers) occupied by various land-use classes.

\begin{tabular}{|l|c|c|c|c|c|c|c|c|c|}
\hline \multirow{2}{*}{ Tipo } & \multicolumn{3}{|c|}{ Total cuenca } & \multicolumn{3}{c|}{ Franja norte } & \multicolumn{3}{c|}{ Franja sur } \\
\cline { 2 - 10 } & 1966 & 2003 & $\Delta \%$ & 1966 & 2003 & $\Delta \%$ & 1966 & 2003 & $\Delta \%$ \\
\hline Agua & 313 & 422 & $\mathbf{2 5 , 8 9}$ & 55 & 94 & $\mathbf{4 1 , 3 3}$ & 19 & 44 & $\mathbf{5 6 , 4 5}$ \\
Urbano-suelo & & & & & & & & & \\
desnudo & 30 & 1.024 & $\mathbf{9 7 , 0 4}$ & 5 & 179 & $\mathbf{9 7 , 4 6}$ & 5 & 116 & $\mathbf{9 5 , 9 4}$ \\
Pastizal-matorral & 11.956 & 14.050 & $\mathbf{1 4 , 9 1}$ & 5.475 & 4.018 & $\mathbf{- 3 6 , 2 5}$ & 2.251 & 3.460 & $\mathbf{3 4 , 9 4}$ \\
Forestal & 20.127 & 23.452 & $\mathbf{1 4 , 1 8}$ & 4.866 & 7.062 & $\mathbf{3 1 , 1 0}$ & 2.388 & 2.922 & $\mathbf{1 8 , 2 6}$ \\
Cultivos & 46.482 & 39.932 & $\mathbf{- 1 6 , 4 0}$ & 5.962 & 5.041 & $\mathbf{- 1 8 , 2 6}$ & 5.106 & 3.235 & $\mathbf{- 5 7 , 8 3}$ \\
\hline
\end{tabular}

\subsection{El cambio hidrológico}

En la Figura 5 se muestran las tendencias en las aportaciones anuales y mensuales durante el periodo de estudio. Nótese que en este caso se presentan los meses según el año hidrológico, con comienzo en octubre y fin en septiembre. Un total de 38 estaciones (casi el 68\%) muestra tendencias anuales negativas estadísticamente significativas, mientras que en 14 estaciones, los valores de tendencia negativa no son significativos. La última estación de aforos del Duero registra una tendencia regresiva significativa, así como las esta- 
ciones del Esla, el Tormes, y el río Águeda que quedan aguas abajo, por lo que se puede afirmar que los recursos hídricos superficiales de la cuenca han experimentado un notable descenso durante el periodo de estudio. Mensualmente se observa que el descenso de los aportes fluviales no es homogéneo. Los meses centrales del invierno y la primavera, que son por su parte los más caudalosos del año, son en los que un mayor porcentaje de estaciones registran tendencias negativas, siendo especialmente numerosas en los meses de febrero y abril. Volviendo a las tendencias mensuales en la precipitación, recordamos que éstas tan sólo eran negativas en los meses de febrero y en menor medida en marzo, lo cual ayudaría a explicar las tendencias hidrológicas en estos meses; sin embargo las precipitaciones de abril mostraban tendencias ligeramente positivas. El descenso de las aportaciones fluviales en el mes de abril puede atribuirse a varias razones. En primer lugar, esto puede ser consecuencia de la inercia que presentan los procesos hidrológicos con respecto a la precipitación, y por ello las tendencias en los caudales de abril todavía reflejan las tendencias en la precipitación de invierno, incluyendo una menor acumulación de nieve durante los meses de febrero y marzo. Por otro lado, podrían existir un factor o factores que estén contribuyendo, a pesar de la precipitación creciente, al descenso en los caudales de primavera, como podría ser el aumento en las temperaturas (y su papel sobre la fusión nival) o el propio incremento de la cobertura vegetal, como se demostrará más adelante. En los meses de verano y otoño, la variabilidad es mayor, aunque el curso principal sigue registrando la inercia de la tendencia negativa registrada en los meses previos.

En la Figura 6 se representa la evolución de las aportaciones promedio de todas las estaciones de aforo (arriba, izquierda) y la de los tramos más caudalosos con estación de aforo (los ríos Duero, Esla y Eresma), apreciándose en ambas que la tendencia regresiva no ha sido constante a lo largo de los años. Parece existir un patrón común de evolución con un descenso paulatino durante los primeros 15 años, un repentino incremento en 1975 que dura 3 años, seguido de otro periodo largo de descenso desde 1978 hasta mediados de los 90. En los últimos años, aunque la evolución es muy variable, parece haber una leve recuperación. El sombreado gris representa el rango intercuartil (es decir el rango que cubre entre el $25 \%$ y el $75 \%$ de los casos), con filtrado de media móvil de 5 años. Éste nos permite observar un periodo de descenso desde el inicio de la serie hasta mediados de los 90, interrumpido por el incremento de principios de los 70 . Se aprecia además con más claridad el ascenso que se produce durante los últimos 15 años de la serie. A pesar de los ciclos existentes, la tendencia general durante el periodo de estudio es decreciente. Mediante un ajuste lineal a las series hidrológicas podemos obtener un valor aproximado del descenso que se ha producido durante el periodo de 

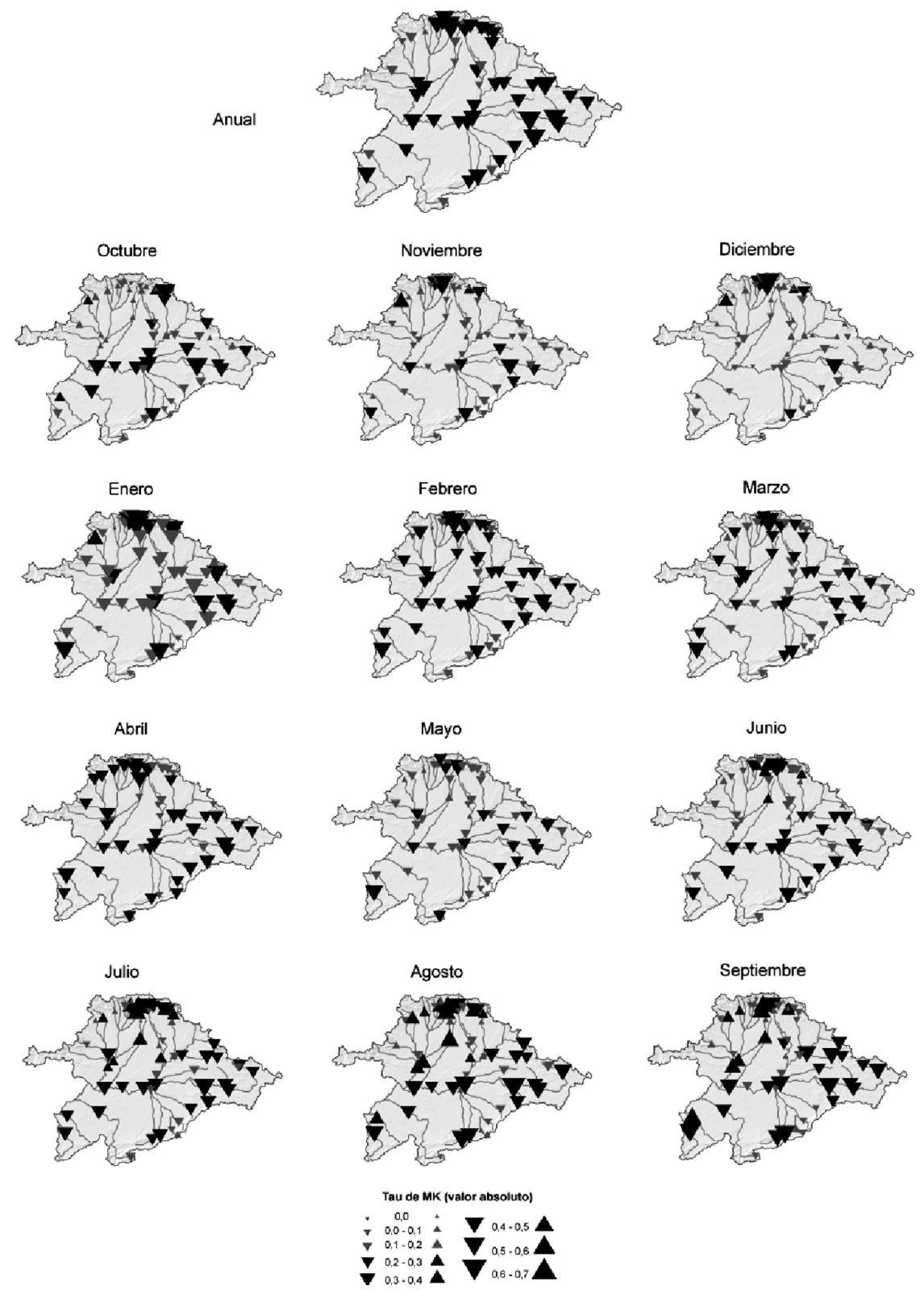

Figura 5. Tendencias en las aportaciones fluviales anuales y mensuales durante el periodo de estudio.

Figure 5. Trends in monthly and annual river flows during the studied period. 
estudio, restando el valor predicho por la recta de ajuste al final de la serie del valor predicho al comienzo. En el río Duero, las pérdidas habrían sido superiores al $120 \%$, pasando de unas aportaciones de $5.230 \mathrm{hm}^{3}$ al comienzo del periodo, a las $2.303 \mathrm{hm}^{3}$ de final del periodo. Algo similar ha ocurrido en los otros dos ríos más caudalosos, el Esla, con un descenso de un $48 \%$, y el Eresma con pérdidas cercanas al 100\%. Sumando las aportaciones del Esla y el Eresma, -que tributan al Duero aguas abajo del aforador estudiado- a las del propio Duero, contaríamos unos aportes de casi $15.000 \mathrm{hm}^{3}$ a comienzos de la serie, y de casi $8.200 \mathrm{hm}^{3}$ a finales del periodo, lo que supone unas pérdidas de casi la mitad de los recursos hídricos en tan sólo medio siglo.
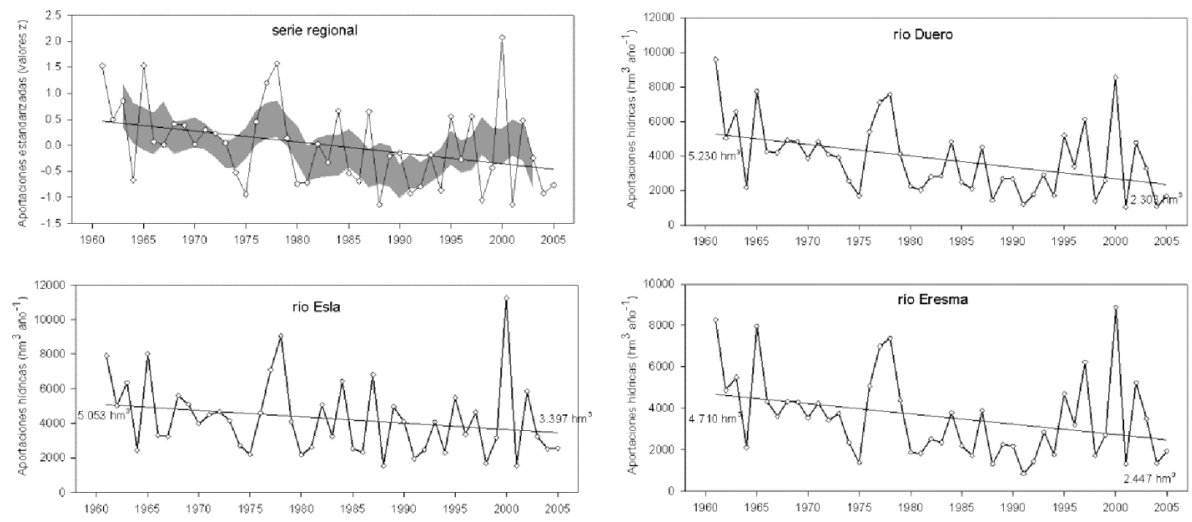

Figura 6. Evolución de las aportaciones fluviales medias de la cuenca (arriba, izquierda) y de los tramos más caudalosos. El sombreado gris representa el rango intercuartil de los casos de estudio, con una media móvil de 5 años.

Figure 6. Evolution of river flows for the average series of the basin (upper-left panel) and in the three main courses of the basin. Grey shade represents the interquartil range of the studied cases with a moving average of 5 years.

El descenso neto de los recursos hídricos no es el único cambio relevante en la hidrología de la cuenca. Mediante un análisis de componentes principales se han caracterizado en la cuenca tres tipos de regímenes fluviales. Un régimen pluvial, localizado en los cursos medios y bajos de los ríos, también en alguna cabecera; un régimen nivo-pluvial, localizado en las zonas más altas de la cuenca; y un régimen alterado por la regulación fluvial. Si nos atenemos a los cursos con régimen natural, observamos en la Figura 7 los cambios que se han producido en los mismos entre la primera y segunda mitad del periodo de estudio en dos ejemplos de estaciones con cada tipo de régi- 
men. En los cursos con régimen pluvial se aprecia que el pico de caudal invernal se localiza en el mes de febrero durante la primera mitad del periodo de estudio. El cambio más notable que se observa en la segunda mitad del periodo de estudio es el brusco descenso de este pico, con el consiguiente retroceso de máximo fluvial al mes de enero. Por su parte en los casos de cursos con régimen nivo-pluvial, además del retroceso de los caudales en el mes de febrero, se produce también un descenso muy notable en el pico nival. Este máximo en la primera mitad del periodo se producía en el mes de abril o mayo, y en la segunda mitad, además de haber perdido magnitud, se ha adelantado también un mes. Además, en uno de los casos mostrados (arriba, derecha) vemos la transformación de un régimen natural en un régimen regulado, con la construcción de un embalse cuya gestión evidencia la retención de agua durante el invierno y la primavera, para ser desembalsada en el verano (de ahí los altos caudales de verano en la segunda mitad del periodo).

Los resultados mostrados hasta ahora evidencian un descenso claro y de gran magnitud de los caudales de la cuenca. Las precipitaciones por su parte no muestran tendencias tan evidentes, por lo que se manifiesta un claro desajuste entre la evolución climática y la hidrológica.
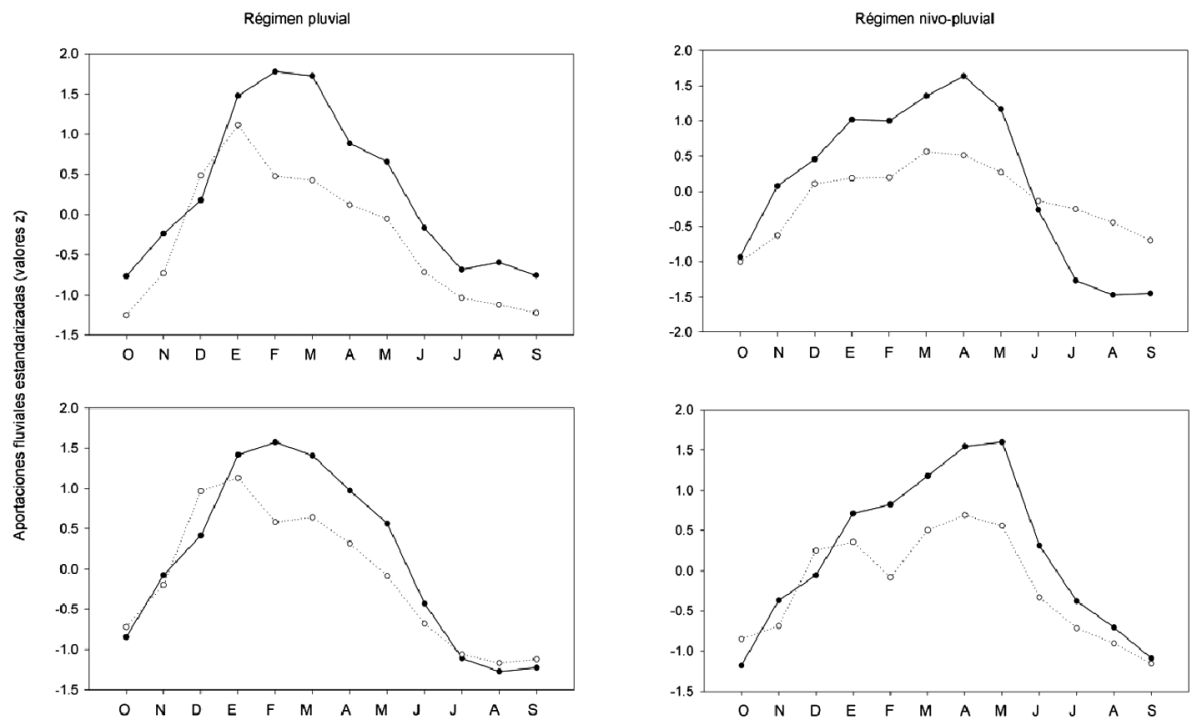

Figura 7. Ejemplos de cambio en los regímenes fluviales entre 1961-83 (línea negra) y 1984-05 (línea gris punteada).

Figure 7. Examples of change of fluvial regimes between 1961-83 (black line) and 1984-05 (grey dotted line). 
Para inferir el papel que las variables climáticas han tenido sobre la evolución hidrológica se han desarrollado regresiones lineales múltiples con las precipitaciones y temperaturas como variables predictoras, para una selección de cuencas de cabecera, en las que la intervención humana ha sido más bien escasa (Beguería et al., 2003, López-Moreno et al. 2011). Las series de precipitaciones y temperaturas introducidas en las regresiones se han obtenido de la siguiente forma: con las 214 series de precipitación y 57 de temperatura se han construido grids climáticos con una resolución 100x100m mediante el método de interpolación explicado en Ninyerola et al. (2000). Para cada cuenca de cabecera se ha obtenido entonces una serie promedio de precipitación y otra de temperatura, que son las que se han utilizado para modelizar la evolución de las aportaciones. El método de regresión "paso a paso" permite introducir las variables en el modelo tan sólo si presentan una contribución estadísticamente significativa $(\alpha<0,05)$ a la explicación de la variable dependiente, y son descartadas si no cumplen con dicho criterio estadístico. En la Tabla 4 se muestran los resultados de dichas regresiones. En primer lugar hay que destacar que la principal variable que explica la evolución de las aportaciones es, en 20 de los 21 casos, la precipitación. Las temperaturas, al contrario de lo que cabría esperar dado su papel sobre la evapotranspiración, tan sólo intervienen de manera significativa en 7 de los modelos realizados. El dato más relevante de la tabla son los coeficientes de MK calculados para los residuales de los modelos, que expresan la tendencia teórica de la parte no explicada por el modelo. En la mayoría de los modelos los residuales presenta coeficientes negativos, aunque tan sólo en 7 casos, las tendencias son estadísticamente significativas. Una tendencia negativa en los residuales indica que las aportaciones están evolucionando con independencia del clima, o dicho de otra forma, que un factor que no ha sido incluido en los modelos está contribuyendo al descenso de las aportaciones. En cuencas de cabecera no reguladas, el único factor capaz de explicar tal descenso es el incremento de la cubierta vegetal, que como se ha demostrado anteriormente ha sido notable en las zonas de montaña de la cuenca.

Mediante un análisis de componentes principales se han identificado dos grupos de estaciones (de entre las estaciones de cabecera seleccionadas) en función de la evolución de los aportes fluviales. Las series agregadas de aportaciones de ambos grupos se muestran con una curva negra en la Figura 8, donde también se muestra la localización de las estaciones pertenecientes a cada grupo o componente principal. Salvo excepciones, la mayoría de estaciones pertenecientes al grupo 1 se encuentran en las cabeceras del norte de la cuenca, mientras que las del grupo 2 se encuentran en las montañas del sur. $\mathrm{Al}$ igual que para las series individuales, se ha modelizado la evolución de las aportaciones con las series climáticas agregadas de cada uno de los compo- 


\section{ENRIQUE MORÁN TEJEDA}

Tabla 4. Resultados de las regresiones lineales realizadas para estimar la evolución de las aportaciones en función de la precipitación y la temperatura, y coeficientes de Mann-Kendall para los residuales de los modelos. Se muestran los coeficientes de cada variable independiente, el porcentaje de varianza explicada $\left(\mathrm{R}^{2}\right)$ y su nivel de significación estadística en el modelo $(\alpha)$. ${ }^{*}$ indica significación estadística $(\alpha<0,05)$.

Table 4. Results of linear regressions for predicting the evolution of streamflows as a function of precipitation and temperature, and Mann-Kendall coefficients for the values of residuals on time. * Indicates significant with a $95 \%$ of confidence.

\begin{tabular}{|c|c|c|c|c|c|c|c|c|c|c|}
\hline \multirow[b]{2}{*}{ Estación } & \multirow[b]{2}{*}{ Constante } & \multicolumn{3}{|c|}{ Precipitación } & \multicolumn{3}{|c|}{ Temperatura } & \multirow[b]{2}{*}{$\begin{array}{c}R^{2} \\
\text { total }\end{array}$} & \multicolumn{2}{|c|}{ Residuales } \\
\hline & & Coef. & $R^{2}$ & $\alpha$ & Coef. & $R^{2}$ & $a$ & & $\begin{array}{l}\text { Tau } \\
M K\end{array}$ & $\alpha$ \\
\hline 2000 & $4,50 \mathrm{E}-11$ & 0,72 & $0,52^{*}$ & 0,00 & - & - & 0,56 & 0,52 & $-0,10$ & 0,32 \\
\hline 2006 & $-3,90 \mathrm{E}-11$ & 0,73 & $0,54^{*}$ & 0,00 & - & - & 0,43 & 0,54 & $-0,22 *$ & 0,03 \\
\hline 2009 & $-1,60 \mathrm{E}-11$ & - & - & 0,14 & - & - & 0,38 & 0,01 & $-0,20$ & 0,05 \\
\hline 2019 & $1,40 \mathrm{E}-11$ & 0,82 & $0,68^{*}$ & 0,00 & 0,21 & $0,04^{*}$ & 0,02 & 0,72 & $-0,17$ & 0,11 \\
\hline 2024 & $-1,90 \mathrm{E}-11$ & 0,68 & $0,45^{*}$ & 0,00 & 0,23 & $0,05^{*}$ & 0,04 & 0,50 & $-0,14$ & 0,19 \\
\hline 2028 & $-4,40 \mathrm{E}-12$ & 0,57 & $0,23^{*}$ & 0,00 & 0,62 & $0,37^{*}$ & 0,00 & 0,60 & $-0,15$ & 0,15 \\
\hline 2030 & $-4,90 \mathrm{E}-11$ & 0,83 & $0,67^{*}$ & 0,00 & 0,34 & $0,12^{*}$ & 0,00 & 0,79 & $-0,08$ & 0,42 \\
\hline 2035 & $2,00 \mathrm{E}-11$ & 0,81 & $0,66^{*}$ & 0,00 & - & - & 0,38 & 0,66 & $-0,55^{*}$ & 0,00 \\
\hline 2046 & $-5,60 \mathrm{E}-11$ & 0,74 & $0,54^{*}$ & 0,00 & - & - & 0,53 & 0,54 & 0,00 & 0,97 \\
\hline 2047 & 5,20E-11 & 0,71 & $0,51^{*}$ & 0,00 & - & - & 0,84 & 0,51 & $-0,21^{*}$ & 0,05 \\
\hline 2050 & $5,70 \mathrm{E}-11$ & 0,83 & $0,64^{*}$ & 0,00 & 0,3 & $0,09^{*}$ & 0,01 & 0,73 & $-0,19$ & 0,08 \\
\hline 2051 & $3,50 \mathrm{E}-11$ & 0,74 & $0,54^{*}$ & 0,00 & - & - & 0,81 & 0,54 & 0,00 & 0,98 \\
\hline 2052 & $-1,20 \mathrm{E}-10$ & 0,67 & $0,45^{*}$ & 0,00 & - & - & 0,69 & 0,45 & $-0,09$ & 0,37 \\
\hline 2068 & 7,20E-12 & 0,68 & $0,47^{*}$ & 0,00 & - & - & 0,11 & 0,47 & $-0,29^{*}$ & 0,00 \\
\hline 2070 & $3,50 \mathrm{E}-12$ & 0,28 & $0,36^{*}$ & 0,00 & - & - & 0,44 & 0,36 & $-0,03$ & 0,70 \\
\hline 2078 & $-6,80 \mathrm{E}-11$ & 0,49 & $0,28^{*}$ & 0,00 & 0,38 & $0,14^{*}$ & 0,03 & 0,42 & $-0,30$ * & 0,00 \\
\hline 2089 & $-4,10 \mathrm{E}-11$ & 0,81 & $0,61^{*}$ & 0,00 & - & - & 0,37 & 0,65 & $-0,02$ & 0,82 \\
\hline 2101 & $-3,70 \mathrm{E}-11$ & 0,38 & $0,16^{*}$ & 0,00 & - & - & 0,24 & 0,16 & $-0,34^{*}$ & 0,00 \\
\hline 2104 & $-7,30 \mathrm{E}-11$ & 0,78 & $0,61^{*}$ & 0,00 & - & - & 0,76 & 0,61 & $-0,25^{*}$ & 0,02 \\
\hline 2107 & $9,90 \mathrm{E}-11$ & 0,84 & $0,70^{*}$ & 0,00 & 0,17 & $0,03^{*}$ & 0,05 & 0,73 & $-0,10$ & 0,35 \\
\hline 2109 & $1,40 \mathrm{E}-10$ & 0,7 & $0,49^{*}$ & 0,00 & - & - & 0,99 & 0,49 & $-0,02$ & 0,87 \\
\hline
\end{tabular}

nentes. Las series predichas y los residuales se representan asimismo en la Figura 8, con curvas grises y negra (en trazo grueso), respectivamente. En ambos casos se aprecia como los residuos presentan una evolución descendente, revelando que las aportaciones observadas descienden en el tiempo a un ritmo superior a las predichas en función del clima, lo cual indica la existencia de un factor con una componente temporal que está influyendo en ese descenso de las aportaciones. El hecho de encontrar una tendencia regresiva más acentuada en los residuos del componente 1 (norte de la cuenca), donde la expansión de la cubierta vegetal ha sido de mayor magnitud, sugiere que 
IMPACTOS RECIENTES DE LOS CAMBIOS AMBIENTALES EN LOS RECURSOS HÍDRICOS...

el crecimiento forestal es el factor que ayuda a explicar el descenso de las aportaciones.

Según dicha hipótesis, en aquellas cuencas donde el incremento de la superficie forestal ha sido mayor, los residuales de los modelos deben presentar una tendencia regresiva más acusada. En el gráfico de dispersión de la Figura 9 se aprecia una relación lineal en esa dirección, si bien alguna cuenca se aleja de la recta de ajuste, dando lugar a un coeficiente de determinación de Pearson $\left(R^{2}\right)$, aunque significativo, no muy elevado.
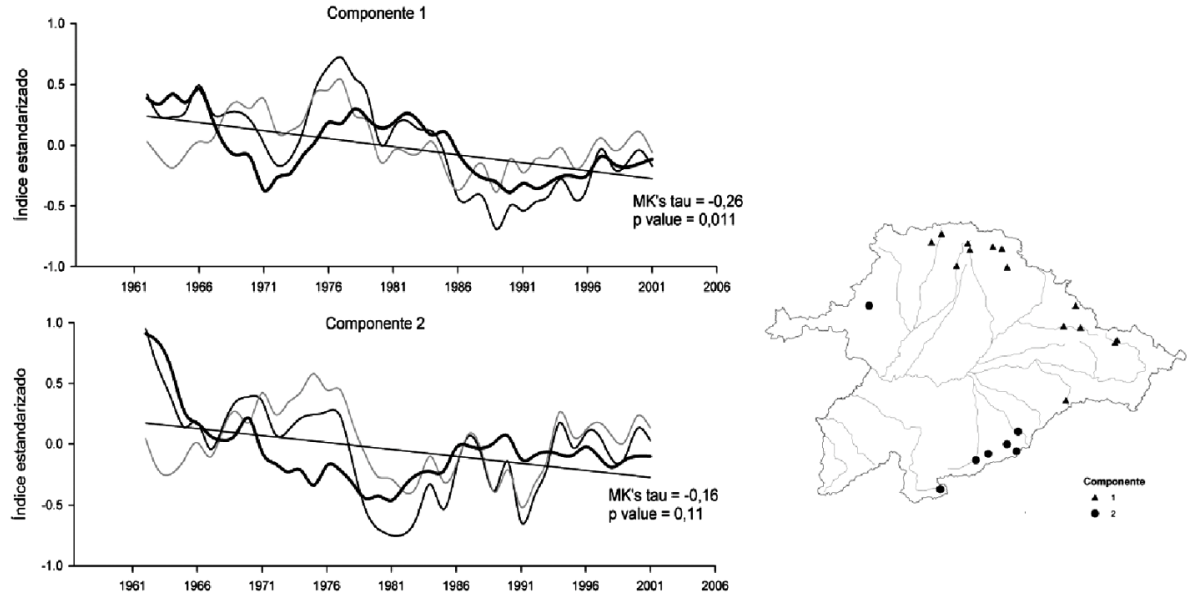

Figura 8. Aportaciones observadas (curva negra fina), predichas (curva gris) y residuos (curva negra, gruesa) para las series agregadas de cada uno de los componentes principales, suavizadas mediante media móvil de 5 años. El ajuste lineal (recta), y los valores de Mann-Kendall corresponden a la evolución de los residuos.

Figure 8. Observed river flows (black thin line), predicted river flows (grey line) and residuals (black thick line) for the aggregated series of each principal component. The linear trend and Mann-Kendall values correspond to the evolution of residuals. 
ENRIQUE MORÁN TEJEDA

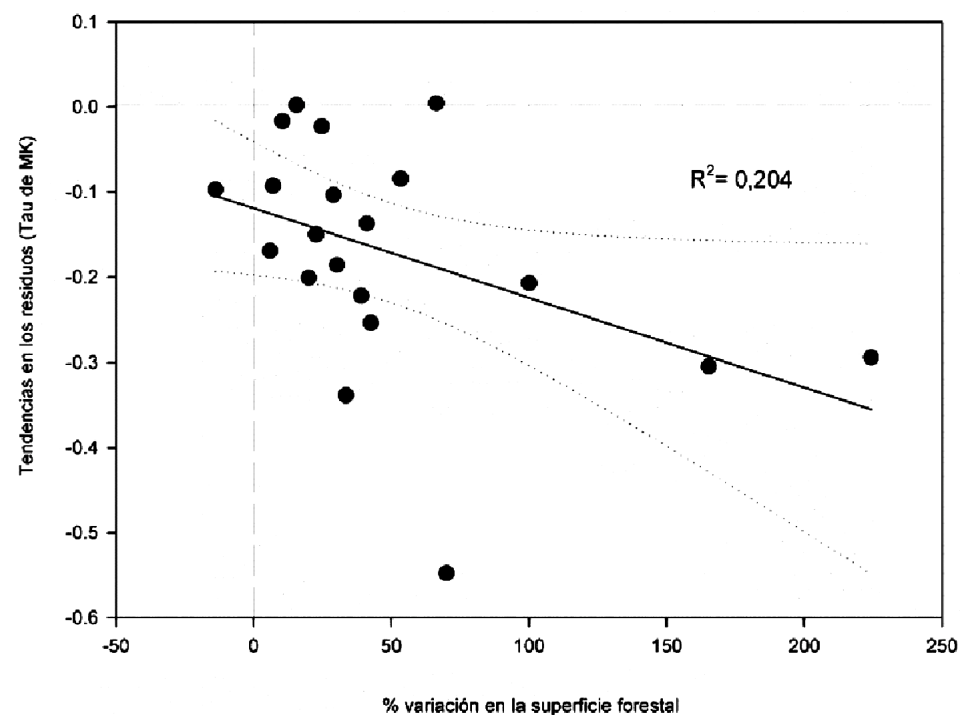

Figura 9. Relación entre los residuales de los modelos y la variación en la superficie forestal. Figure 9. Relationship between residuals of models and variation of forest surface.

\subsection{El papel regulador de los embalses}

Hasta ahora se han mostrado estadísticas de la evolución de los recursos hídricos en régimen natural, o escasamente alterado por el hombre. A continuación se muestran unos ejemplos de cómo la gestión de algunos embalses modifica no sólo el régimen fluvial sino la disponibilidad de agua a largo plazo, en función de los patrones de gestión. En la Figura 10 se representa el régimen fluvial (promedio para el periodo de estudio) a la entrada y salida de 4 embalses de la cuenca, así como las reservas mensuales en los mismos. Se han seleccionado estos 4 ejemplos, ya que ilustran los modelos de gestión tipo que se dan en la cuenca: por un lado observamos un modelo en el que apenas se modifica el régimen fluvial (Cervera-Ruesga), tan sólo se retienen pequenas cantidades de agua en el invierno que sirven para aumentar las reservas en la primavera y el verano. El valor de correlación $(R=0,95)$ entre las entradas y salidas mensuales indica que apenas se produce modificación del régimen fluvial. En segundo lugar se observa un patrón en el que se retienen mayores cantidades de agua durante el invierno (La Requejada), con la consecuente modificación del régimen fluvial $(R=0,24)$. Y por último encontramos dos ejemplos (Barrios de Luna y Cuerda del Pozo) en los que la retención 
de caudales en el invierno es muy fuerte, y éstos son posteriormente liberados en el verano para abastecer diferentes demandas (riego, abastecimiento urbano, etc.). En estos casos se produce una inversión del régimen fluvial, de ahí los valores negativos de correlación entre entradas y salidas $(R=-0,14$ y $-0,67$ respectivamente).
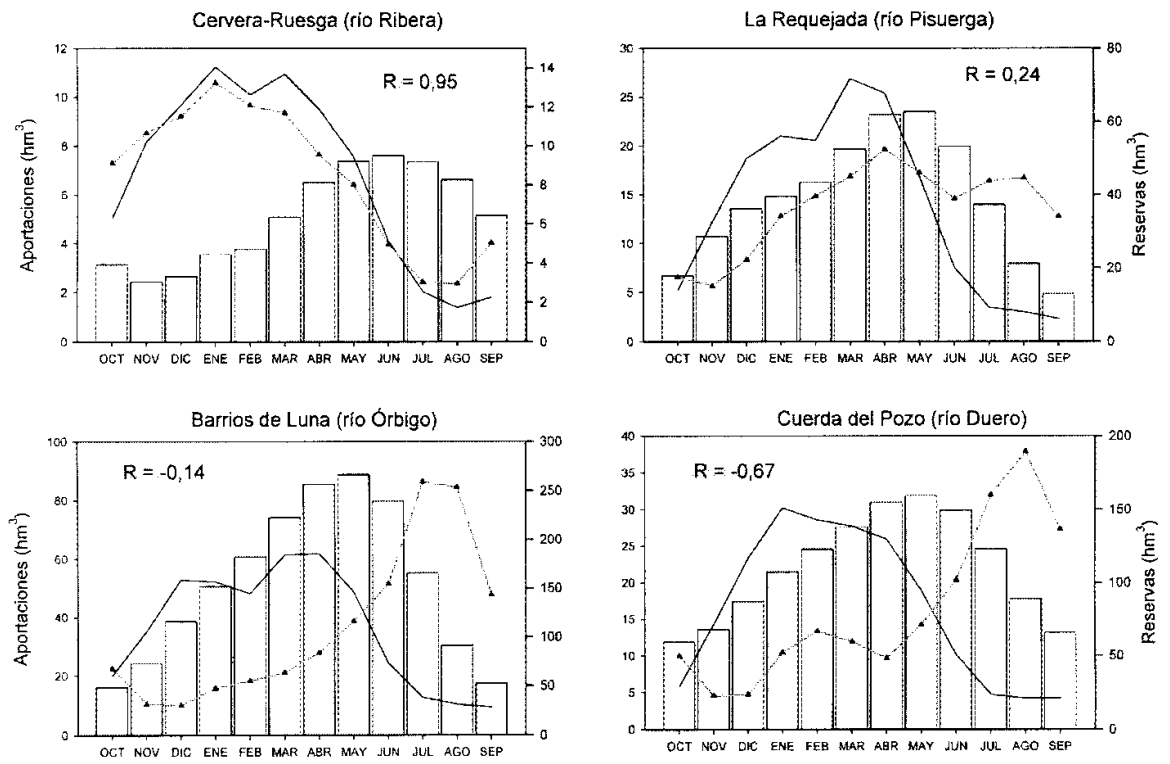

Figura 10. Entradas (línea negra), salidas (línea gris) y reservas (barras) mensuales en cuatro embalses de la cuenca del Duero, promediadas para el periodo de estudio. R: coeficiente de correlación de Pearson.

Figure 10. Inflows (black line), outflows (grey line) and volume of water storage (bars) in 4 reservoirs of the basin. R: Pearson's correlation coefficient.

El coeficiente de correlación de Pearson informa del nivel de regulación o alteración del régimen fluvial por parte de los embalses. Mientras que la Figura 10 presenta una imagen estática de dicho nivel de regulación promediado durante el periodo de estudio, en la Figura 11 podemos ver la evolución del mismo a lo largo de los años. Tanto para los embalses cuyo coeficiente de correlación medio indica una leve regulación, como para aquellos en los que la alteración del régimen es elevada, durante las últimas décadas se aprecia una ligera tendencia hacia un mayor nivel de regulación, esto es, hacia valores más bajos del coeficiente de correlación. Este aumento de la alteración fluvial ha ido acompañado de un incremento sostenido de los niveles de embalsado, sobre todo en los dos embalses con mayor nivel de regulación, 
Barrios de Luna y Cuerda del Pozo, como se aprecia en la Figura 11. En este sentido, cabe destacar la diferencia en la evolución de los niveles de embalsado entre ambos, a pesar de mostrar una tendencia del mismo signo. En el embalse de Cuerda del Pozo el incremento del nivel de embalsado se produce con una notable variabilidad inter-anual, lo cual sugiere que se ha ido adaptando el nivel de embalsado a las demandas o bien a la disponibilidad de agua que gobierna la variabilidad climática. Por el contrario en el embalse de Barrios de Luna se observa cómo los niveles de embalsado se han mantenido constantes durante periodos largos de tiempo, y en momentos puntuales se han bajado o incrementado, dando lugar a un balance final de incremento de los niveles. Este patrón indica más una gestión que depende de los intereses particulares de los gestores del embalse, por encima de otras consideraciones como la disponibilidad o demanda de agua.

En cualquier caso se observa que los ríos están experimentando cada vez niveles más altos de regulación, a costa de aumentar los embalses las reservas a lo largo de los años, de lo que se deduce igualmente que los embalses están contribuyendo al descenso de los caudales de los ríos de la cuenca.
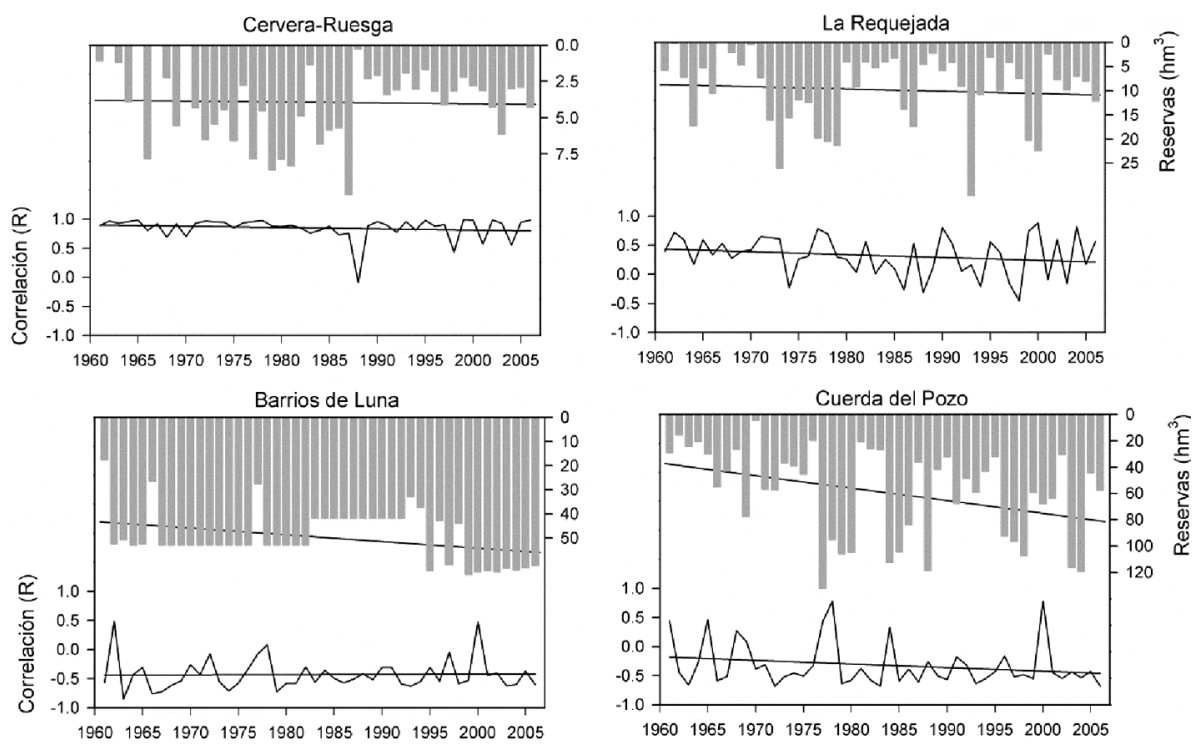

Figura 11. Evolución del coeficiente de correlación entre entradas y salidas mensuales (curva negra) y del volumen de agua embalsada (barras grises) durante el periodo de estudio. Las líneas rectas se corresponden con ajustes lineales para ilustrar la tendencia temporal. Nótese que el eje correspondiente a las reserves (derecha) tiene los valores invertidos.

Figure 11. Evolution of the correlation coefficient between inflows and outflows (black line) and evolution of the water storage (grey bars) during the studied period. Straightlinesindicate linear trends. 
IMPACTOS RECIENTES DE LOS CAMBIOS AMBIENTALES EN LOS RECURSOS HÍDRICOS...

\section{Discusión y conclusiones}

Los recursos hídricos de la cuenca del Duero han experimentado un retroceso notable durante la segundo mitad del siglo XX. En este trabajo, además de poner de manifiesto las características de este retroceso, se evalúan las que pensamos que son las principales causas del mismo.

De forma general se puede atribuir el descenso global de los recursos hídricos a la reducción de las aportaciones fluviales en invierno y primavera, coincidiendo con los momentos de máximo caudal. En los ríos con máximo invernal el descenso es mayor en los meses de febrero, resultando en un desplazamiento del pico a meses anteriores. Otra característica común es el retroceso significativo de los caudales de primavera, que implica también el adelantamiento en un mes del pico primaveral. Asistimos por lo tanto a dos procesos complementarios, que a simple vista deberían implicar una preocupación en los gestores de los recursos hídricos ya que se cuenta con menos agua para abastecer una demanda que a priori no ha descendido (Gil Olcina 1999). A ello hay que añadirle la incertidumbre derivada de las causas de dichos cambios, ya que, como se ha comprobado en este trabajo, y se discute a continuación, pueden ser consecuencia de numerosos procesos, alguno de los cuales seguramente se escape de los contenidos de este estudio.

En primer lugar el descenso y redistribución mensual de los caudales se debe explicar, como no podría ser de otra forma, desde el punto de vista de la evolución climática de la cuenca. El clima es el factor responsable de las entradas, y parte de las salidas, de agua en las cuencas hidrográficas. Si hay una característica que defina la evolución climática en la Península Ibérica, esta es la variabilidad (Bladé \& Castro-Díez, 2010). Las precipitaciones, y en menor medida las temperaturas, en la región mediterránea, presentan una evolución variable en el tiempo. A esta incertidumbre climatológica se ha unido históricamente el conflicto entre disponibilidad y demanda, ya que normalmente el consumo de las mayores cantidades de agua se produce en las épocas en que la disponibilidad es menor (Iglesias, 2005). No obstante, superpuestas a esta variabilidad, en muchas ocasiones se encuentran tendencias a largo plazo en las variables climáticas, que pueden condicionar en mayor medida la disponibilidad de los recursos hídricos. En el caso de las precipitaciones, si bien observamos que han descendido en el mes de febrero, no muestran por lo general descensos significativos. De hecho las series anuales de precipitación se podría decir que no han experimentado tendencias claras de ningún signo. Nuestras observaciones no contradicen en exceso los resultados obtenidos en la mayoría de los trabajos realizados sobre la evolución de las precipitaciones a lo largo del siglo XX en el sur de Europa. Aunque los estudios suelen diferir en cuanto a periodos de tiempo analizados, escala 
espacial del análisis, número de observatorios, o incluso tests estadísticos utilizados, en general no se ha podido detectar un descenso significativo de las precipitaciones en la región (Giorgi, 2002, Norrant \& Douguedroit, 2006, Gonzalez-Hidalgo et al., 2010). Por el contrario, la evolución de las temperaturas en la región muestra un claro signo de incremento, siendo las tendencias más evidentes en los meses de diciembre, y entre la primavera y el verano. El aumento térmico demostrado para la cuenca del Duero coincide con el incremento general registrado en todo el territorio peninsular, cuyo origen se cifra por la mayoría de los autores a comienzos de la década de los 70 (De Castro et al., 2005, Brunet et al., 2007, Bladé \& Castro-Díez, 2010). Asimismo, el análisis por regiones mundiales que ofrece el último informe del Panel Intergubernamental sobre Cambio Climático (IPCC 2007) muestra para el continente europeo un punto de inflexión a comienzos de la década de los 70, a partir del cual las temperaturas aumentan de forma sostenida hasta la actualidad, y pronostica una continuidad en el incremento térmico a lo largo del siglo XXI, como causa del aumento en la concentración atmosférica de gases invernadero de origen antrópico.

Volviendo a los cambios hidrológicos observados, el descenso en los caudales de invierno podría estar reflejando las tendencias negativas en la precipitación de febrero. Sin embargo observamos cómo ya en diciembre y enero se aprecian tendencias significativas en los caudales, las cuales no pueden ser explicadas por la evolución en la precipitación. Por otro lado observamos una caída y retroceso del pico nival en aquellos tramos fluviales en que el máximo se produce en la primavera. Esto se debe probablemente a una combinación de dos procesos. En primer lugar, las tendencias negativas en la precipitación de febrero sugieren un descenso también en la precipitación en forma de nieve, y por lo tanto menor acumulación de nieve en las partes altas de las montañas, lo que a su vez supone una menor cantidad de agua de escorrentía una vez que se produce la fusión del manto nivoso en primavera. En segundo lugar, la ocurrencia más temprana del pico primaveral podría ser atribuida al incremento de las temperaturas de invierno y primavera, lo que provocaría tanto una menor acumulación de nieve, como una fusión más temprana. Este incremento térmico, durante el invierno y la primavera es considerado responsable de cambios en los regímenes fluviales en otros lugares de la península. Por ejemplo, López-Moreno (2005) demostró en los Pirineos una evolución regresiva del manto nivoso de abril durante las últimas cinco décadas, la cual presentaba buena correlación con las tendencias en precipitación en los meses precedentes a la fusión, y con las tendencias de las temperaturas de abril. Más recientemente, Pons et al.,(2009) encontraron tendencias negativas en el número de "días de nieve" durante el invierno y la primavera en la mitad norte de España (incluyendo puntos de medición en la 
cuenca del Duero) durante las últimas tres décadas. En este caso, los autores demostraron que estas tendencias se correlacionaban mejor con la evolución de las temperaturas que con las de las precipitaciones. Cambios en los regímenes fluviales similares a los observados en este trabajo han sido detectados en otras zonas de montaña de la Península Ibérica y de la región Mediterránea. Por ejemplo López-Moreno et al.,(2008) y García-Ruiz et al.,(2001)demostraron una reducción significativa de los caudales primaverales, con una ocurrencia más temprana del pico máximo en los ríos pirenaicos, lo cual atribuyeron a la reducción de la cantidad de nieve acumulada, por condicionantes climáticos. Kalayci and Kahya (2006) encontraron tendencias negativas en los caudales mensuales de distintos ríos de Turquía, y lo relacionaron con las crecientes temperaturas y evapotranspiración.

A pesar de que las variables climáticas pueden explicar en parte el descenso observado en las aportaciones fluviales, encontramos evidencias de una evolución dispar, sobre todo entre caudales y precipitaciones que hace pensar que un factor no climático está contribuyendo al descenso hidrológico. Dada esta disparidad entre la evolución de las precipitaciones y los caudales, nuestra principal hipótesis para explicar el descenso hidrológico radica en el incremento observado de la cubierta vegetal y el papel hidrológico de la misma demostrado en cuencas experimentales. En el presente trabajo se pone de manifiesto que el crecimiento de la cubierta vegetal ha podido tener un papel relevante en el descenso de los aportes fluviales. Tres observaciones verifican dicha hipótesis: (i) existe en todos los casos de estudio una tendencia regresiva en los aportes fluviales más marcada que en las precipitaciones; (ii) Los residuos de los modelos presentan coeficientes de Mann-Kendall negativos, lo cual indica una separación progresiva entre la evolución de las aportaciones y la evolución climática, y (iii) la disparidad entre la evolución de las aportaciones y de las precipitaciones, y las tendencias regresivas en los aportes son más evidentes en las cabeceras fluviales localizadas en el norte de la cuenca, donde la expansión del bosque y el matorral de montaña ha afectado a mayores áreas. Tomando esta relación como hipótesis principal distintos investigadores, a partir de métodos estadísticos, han estimado el papel de los cambios en los usos del suelo sobre la evolución interanual de los caudales en grandes cuencas hidrológicas o conjuntos regionales. Por ejemplo, Begueríaet al., (2003), Gallart\& Llorens (2004), y López-Moreno et al.,(2011) comprueban para un conjunto de cabeceras fluviales tributarias del Ebro una separación sistemática de la evolución de las aportaciones respecto a las precipitaciones, así como una tendencia significativa en los residuos, que achacan al crecimiento de la cubierta vegetal en las cuencas de drenaje, y lo cuantifican en un $30 \%$ y un $17 \%$ respectivamente. También Gallart \& Llorens (2002) perciben un importante descenso en los aportes del Ebro en su desem- 
bocadura en Tortosa, de los cuales atribuyen un tercio al descenso en las precipitaciones, otro tercio al consumo por los regadíos, y un tercio también al crecimiento en la cubierta forestal en las cabeceras fluviales.

Un resultado relevante de nuestros análisis es la variable, pero en general escasa, participación de la "temperatura" en los modelos para explicar la evolución de las aportaciones hídricas. Desde un punto de vista teórico las crecientes temperaturas deberían estar provocando un aumento en la evapotranspiración y afectando negativamente al balance hídrico de las cuencas; que esto no se vea reflejado en los modelos se debe probablemente a la escala de análisis utilizada. El hecho de trabajar con datos anuales puede estar enmascarando procesos significativos a escala estacional, pero no suficientemente relevantes a escala anual. Anteriormente se apuntó a la posible influencia de las crecientes temperaturas sobre los cambios en los regímenes fluviales, sin embargo su papel tendría más que ver con el adelanto del pico primaveral de origen nival que con el descenso neto de las aportaciones anuales. Lespinas et al. (2010), argumentan que el descenso en los caudales de ríos de montaña del sur de Francia está asociado con el papel de las crecientes temperaturas en el control de la acumulación y fusión de la nieve, sin embargo no consideró el posible impacto de los cambios en los usos del suelo ocurridos en su zona de estudio. La evapotranspiración es un proceso dependiente de la actividad vegetativa de las plantas, y el consumo de agua por parte de éstas se produce básicamente durante la primavera y el verano. Un aumento de las temperaturas significaría un incremento potencial de la necesidad hídrica de las plantas y por tanto mayor consumo sobre todo en los meses de verano, cuando el estrés hídrico es más notable. El escaso peso que representan las aportaciones de verano con respecto al total anual $(<5 \%)$, explicaría que este proceso no se vea reflejado sobre la evolución interanual de las aportaciones hídricas. En cualquier caso, el papel de los cambios en la cobertura vegetal y del aumento en las temperaturas deben estar íntimamente relacionados a través del proceso de evapotranspiración, y resultan extremadamente complejos de separar en base a estudios estadísticos.

Por último, se ha mostrado la capacidad de algunos embalses para modificar las condiciones hidrológicas, y se atisba una tendencia hacia mayores niveles de regulación y embalsado, con el consiguiente descenso de los caudales, aguas abajo de los mismos. Distintos estudios realizados hasta la fecha han comprobado igualmente la capacidad de los embalses para modificar los regímenes fluviales aguas abajo de la presa. En nuestro ámbito más cercano, el trabajo de López-Moreno (2006) demuestra que los embalses pirenaicos presentan, por lo general, modificaciones del régimen fluvial más suaves que los embalses de la cuenca del Duero, y raramente se observan inversiones del régimen fluvial como las demostradas en este trabajo. Por otro lado, Batalla et 
al. (2004), estudiaron los cambios hidrológicos producidos por los embalses de la cuenca del Ebro y observaron patrones de modificación de los regímenes fluviales similares a los demostrados en este trabajo. Así, mientras unos embalses apenas modificaron la distribución estacional de los caudales aguas abajo, otros, principalmente destinados al riego durante el verano, producían una inversión total del régimen. En función de nuestras observaciones podemos argumentar que la política hidrológica de la cuenca está apostando por una gestión de los recursos en su oferta a través del incremento de las reservas para hacer frente a una disponibilidad de agua cada vez menor. Una política hidrológica basada en el aprovisionamiento de recursos será a corto plazo insostenible, en caso de que continúe el descenso de caudales en la cuenca observado en este trabajo, acorde con las previsiones de evolución climática para las próximas décadas (Iglesias, 2005).

La disponibilidad futura de recursos hídricos presenta altos niveles de incertidumbre. Los modelos climáticos predicen un descenso de hasta un $20 \%$ en los recursos hídricos de la región mediterránea durante el curso del siglo presente (IPCC, 2007). Para la cuenca del Duero hemos demostrado que el clima no puede explicar únicamente el descenso en los caudales. Por ello el principal reto que se plantea, derivado de los resultados obtenidos, es la modelización de una de las variables más importantes para explicar las pérdidas de agua, la evapotranspiración. Ésta conjuga tanto condiciones climáticas como condiciones de cubierta vegetal y es por tanto imprescindible su conocimiento a la hora de plantear proyecciones futuras de disponibilidad de agua en escenarios de cambio climático.

Los resultados de este trabajo nos permiten concluir que el descenso hidrológico demostrado no debería ser estudiado solamente desde el ámbito científico. El reconocimiento del mismo por parte de los responsables o gestores públicos del recurso es esencial a corto plazo. Cualquier intervención relativa a la gestión de los recursos hídricos de la cuenca debe ser considerada desde una perspectiva global que considere las sinergias entre las montañas y el llano, lugares respectivos de origen y consumo de los mismos. Solamente una gestión integrada del territorio podría minimizar los impactos del descenso hidrológico sobre los ecosistemas, la población y las actividades económicas de la cuenca del Duero.

\section{Agradecimientos}

El presente estudio constituye una síntesis de los resultados de la tesis doctoral defendida por el autor en la Facultad de Geografía e Historia de la Universidad de Salamanca, en marzo de 2011. El trabajo ha sido posible gra- 
cias una beca de investigación concedida por la Junta de Castilla y León (Becas de formación del personal investigador, orden EDU/918/2006 del 30 de mayo) disfrutada entre los años 2006 y 2010. El autor expresa su más sincero agradecimiento a Antonio Ceballos y Nacho López por la supervisión; y a Sergio Vicente, Javier Zabalza, José Manuel Llorente, Jorge Lorenzo y Santiago Beguería por su colaboración en las distintas etapas de elaboración del trabajo.

\section{Referencias}

Alexandersson, H. 1986. A Homogeneity test applied to precipitation data.Journal of Climatology, 6:661-675.

Beguería, S., López-Moreno, J. I., Lorente, A., Seeger, M. \& García-Ruiz, J. M. 2003. Assessing the effect of climate oscillations and land-use changes on streamflow in the Central Spanish Pyrenees. Ambio, 32:283-286.

Beniston, M. 2005. Mountain climates and climatic change: An overview of processes focusing on the European Alps. Pure and Applied Geophysics, 162:1587-1606. Doi:10.1007/s00024-005-2684-9.

Bent, G. C. 2001. Effects of forest-management activities on runoff components and ground-water recharge to Quabbin Reservoir, central Massachusetts. Forest Ecology and Management, 143:115-129.

Berkun, M. 2010. Hydroelectric potential and environmental effects of multidam hydropower projects in Turkey. Energy for Sustainable Development, 14:320-329.

Berryman, D., Bobee, B., Cluis, D. \& Haemmerli, J. 1988. Nonparametric tests for trend detection in water-quality time series. Water Resources Bulletin, 24:545-556.

Birsan, M. V., Molnar, P., Burlando, P. \& Pfaundler, M. 2005. Streamflow trends in Switzerland. Journal of Hydrology, 314:312-329.

Bladé, I. \& Castro-Díez, Y. 2010. Tendencias atmosféricas en la Península Ibérica durante el periodo instrumental en el contexto de la variabilidad natural. En F. Pérez \& R. Boscolo, (Eds). Clima en España: pasado, presente y futuro. 25-42 pp.

Bonacci, O. \& Roje-Bonacci, T. 2003. The influence of hydroelectrical development on the flow regime of the karstic river Cetina. Hydrological Processes, 17:1-15. Doi:10.1002/hyp.1190.

Bosch, J. M. \& Hewlett, J. D. 1982. A review of catchment experiments to determine the effect of vegetation changes on water yield and evapo-transpiration. Journal of Hydrology, 55:3-23. 
Brunet, M., Jones, P. D., Sigro, J., Saladie, O., Aguilar, E., Moberg, A., DellaMarta, P. M., Lister, D., Walther, A. \& Lopez, D. 2007. Temporal and spatial temperature variability and change over Spain during 1850-2005. Journal of Geophysical Research-Atmospheres, 112. Doi:10.1029/2006jd008249.

Cosandey, C., Andreassian, V., Martin, C., Didon-Lescot, J. F., Lavabre, J., Folton, N., Mathys, N. \& Richard, D. 2005. The hydrological impact of the mediterranean forest: a review of French research. Journal of Hydrology, 301:235-249. Doi:10.1016/j.jhydrol.2004.06.040.

Cosandey, C. \& Robinson, M. 2000. Hydrologie continentale. Armand Colin, Paris, $360 \mathrm{pp}$.

Crockford, R. H. \& Richardson, D. P. 2000. Partitioning of rainfall into throughfall, stemflow and interception: effect of forest type, ground cover and climate. Hydrological Processes,14:2903-2920.

De Castro, M., Martín-Vide, J. \& Alonso, S. 2005. El clima de España: pasado, presente y escenarios de clima para el siglo XXI. pp 1-64 En J. M. C. Moreno Rodríguez, (Ed). Evaluación preliminar de los impactos en España por efecto del cambio climático. Ministerio de Medio Ambiente y Universidad de Castilla-La Mancha.

Gallart, F. \& Llorens, P. 2003. Catchment management under environmental change: Impact of land cover change on water resources. Water International, 28:334-340.

García-Ruiz, J. M., Beguería Portugués, S., López Moreno, J. I., Lorente Grima, A. \& Seeger, M. 2001. Los recursos hidricos superficiales del Pirineo aragonés y su evolución reciente. Geoforma Ediciones, Logroño.

García-Ruiz, J. M., Lasanta, T., Ruiz-Flano, P., Ortigosa, L., White, S., González, C. \& Martí, C. 1996. Land-use changes and sustainable development in mountain areas: a case study in the Spanish Pyrenees. Landscape Ecology, 11:267-277.

García-Ruiz, J. M., López-Moreno, J. I., Vicente-Serrano, S. M., LasantaMartínez, T. \& Beguería, S. 2011. Mediterranean water resources in a global change scenario. Earth-Science Reviews, 105:121-139.

Gil Olcina, A. 1999. Los usos del agua en España: una perspectiva histórica. En A. Gil Olcina \& A. Morales Gil, (Eds). Los Usos del Agua en España. Caja de Ahorros del Mediterráneo e Instituto Universitario de Geografía (Universidad de Alicante), Alicante, pp 13-48.

Giorgi, F. 2002. Variability and trends of sub-continental scale surface climate in the twentieth century. Part I: observations. Climate Dynamics, 18:675-691. Doi:10.1007/ s00382-001-0204-x.

Gonzalez-Hidalgo, J. C., Brunetti, M. \& de Luis, M. 2010. Precipitation trends in Spanish hydrological divisions, 1946-2005. Climate Research, 43:215-228. Doi:10.3354/cr00937. 
Hirsch, R. M., Slack, J. R. \& Smith, R. A. 1982. Techniques of trend analysis for monthly water quality data. Water Resources Research, 18:107-121.

Iglesias, A., Estrela, T., Gallart, F. 2005. Impactos sobre los recursos hídricos. In: Moreno Rodríguez, J.M. (Coord.).Evaluación preliminar de los impactos en España por efecto del cambio climático. Ministerio de Medio Ambiente y Universidad de Castilla-La Mancha. 303-354.

IPCC. 2007. Climate Change 2007: Synthesis Report. [Core Writing Team, Pachauri RK and Reisinger A (eds.)]. IPCC: Geneva, Switzerland, 104 pp. Contribution of Working Groups I, II and III to the Fourth Assessment Report of the Intergovernmental Panel on Climate Change.

Jollife, I. T. 2002. Principal Component Analysis. 2 edition. Springer, New York.

Kahya, E. \& Kalayci, S. 2004. Trend analysis of streamflow in Turkey. Journal of Hydrology, 289:128-144.

Kalayci, S. \& Kahya, E. 2006. Assessment of streamflow variability modes in Turkey: 1964-1994. Journal of Hydrology, 324:163-177.

Kendall, M. G. 1975. Kendall Rank Correlation Methods. Griffin, London.

Kundzewicz, Z. W. \& Robson, A. J. 2004. Change detection in hydrological records-a review of the methodology / Revue méthodologique de la détection de changements dans les chroniques hydrologiques. Hydrological Sciences Journal, 49: 7-19.

Lanzante, J. R. 1996. Resistant, robust and non-parametric techniques for the analysis of climate data: Theory and examples, including applications to historical radiosonde station data. International Journal of Climatology, 16:1197-1226.

Lasanta-Martínez, T., Vicente-Serrano, S. M. \& Cuadrat-Prats, J. M. 2005. Mountain Mediterranean landscape evolution caused by the abandonment of traditional primary activities: a study of the Spanish Central Pyrenees. Applied Geography, 25:47-65. Doi:10.1016/j.apgeog.2004.11.001.

Lehmann, E. L. 1975. Nonparametrics Statistical Methods Based on Ranks. Holden-Day, Inc., California.

Lespinas, F., Ludwig, W. \& Heussner, S. 2010. Impact of recent climate change on the hydrology of coastal Mediterranean rivers in Southern France. Climatic Change, 99:425-456.

Lettenmaier, D. P., Wood, E. F. \& Wallis, J. R. 1994. Hydro-climatological trends in the continental United States, 1948-88. Journal of Climate, 7:586607.

Llorens, P. \& Domingo, F. 2007. Rainfall partitioning by vegetation under Mediterranean conditions. A review of studies in Europe. Journal of Hydrology, 335:37-54. Doi:10.1016/j.jhydrol.2006.10.032.

López-Moreno, J. I. 2005. Recent variations of snowpack depth in the central Spanish Pyrenees. Arctic, Antarctic and Alpine Research, 37:253-260. 
López-Moreno, J. I. 2006. Cambio ambiental y gestión de los embalses en el Pirineo Central español. Consejo de Portección de la Naturaleza de Aragón, Zaragoza, 208 pp.

López-Moreno, J. I., Begueria, S. \& Garcia-Ruiz, J. M. 2002. Influence of the Yesa reservoir on floods of the Aragon River, central Spanish Pyrenees. Hydrology and Earth System Sciences, 6:753-762.

López-Moreno, J. I., Beniston, M. \& García-Ruiz, J. M. 2008. Environmental change and water management in the Pyrenees: Facts and future perspectives for Mediterranean mountains. Global and Planetary Change, 61:300312.

López-Moreno, J. I., Vicente-Serrano, S. M., Morán-Tejeda, E., Zabalza, J., Lorenzo-Lacruz, J. \& García-Ruiz, J. M. 2011. Impact of climate evolution and land use changes on water yield in the Ebro basin. Hydrology and Earth System Sciences, 15:311-322.

Maingi, J. K. \& Marsh, S. E. 2002. Quantifying hydrologic impacts following dam construction along the Tana River, Kenya. Journal of Arid Environments, 50:53-79.

Mann, H. B. 1945. Non parametric test against trend. Econometrica, 13:245-259.

Milly, P. C. D., Dunne, K. A. \& Vecchia, A. V. 2005. Global pattern of trends in streamflow and water availability in a changing climate. Nature, 438:347350.

Morán, C. 2007. Trascolación en un bosque de roble melojo (Quercus pyrenaica willd) en la sierra de Tamames (Salamanca). Trabajo de Grado Inédito. Universidad de Salamanca.

Nilsson, C., Reidy, C. A., Dynesius, M. \& Revenga, C. 2005. Fragmentation and flow regulation of the world's large river systems. Science, 308:405408. Doi:10.1126/science.1107887.

Ninyerola, M., Pons, X. \& Roure, J. M. 2000. A methodological approach of climatological modelling of air temperature and precipitation through GIS techniques. International Journal of Climatology, 20:1823-1841.

Norrant, C. \& Douguedroit, A. 2006. Monthly and daily precipitation trends in the Mediterranean (1950-2000). Theoretical and Applied Climatology, 83:89-106. Doi:10.1007/ s00704-005-0163-y.

Peterson, T. C., Easterling, D. R., Karl, T. R., Groisman, P., Nicholls, N., Plummer, N., Torok, S., Auer, I., Boehm, R., Gullett, D., Vincent, L., Heino, R., Tuomenvirta, H., Mestre, O., Szentimrey, T., Salinger, J., Forland, E. J., Hanssen-Bauer, I., Alexandersson, H., Jones, P. \& Parker, D. 1998. Homogeneity adjustments of in situ atmospheric climate data: A review. International Journal of Climatology, 18:1493-1517.

Petts, G. E. 1984. Impounded rivers. John Willey \& sons Eds, Chichester, 326 pp. 
Pons, M. R., San-Martín, D., Herrera, S. \& Gutiérrez, J. M. 2009. Snow trends in Northern Spain: analysis and simulation with statistical downscaling methods. International Journal of Climatology, 30:1795-1806.

Poyatos, R., Latron, J. \& Llorens, P. 2003. Land use and land cover change after agricultural abandonment - The case of a Mediterranean Mountain Area (Catalan Pre-Pyrenees). Mountain Research and Development, 23:362368.

Rodríguez-Puebla, C., Encinas, A. H., Nieto, S. \& Garmendia, J. 1998. Spatial and temporal patterns of annual precipitation variability over the Iberian Peninsula. International Journal of Climatology, 18:299-316.

Sneyers, R. 1990. On the statistical analysis of series of observations. Technical Note - World Meteorological Organization, Geneva.

Tabachnick, B. \& Fidell, L. 1996. Using multivariate statistics. 3 edition. Harper Collins, New York.

Verstraeten, G. \& Poesen, J. 2000. Estimating trap efficiency of small reservoirs and ponds: methods and implications for the assessment of sediment yield. Progress in Physical Geography, 24:219-251.

Vicente-Serrano, S., Beguería, S. \& Lasanta-Martínez, T. 2000. El proceso de revegetación en un área de montaña como consecuencia de los cambios de gestión: Aplicación de un SIG al Valle Borau (Pirineo Central Español).En:Tecnologías Geográficas para el Desarrollo Sostenible, pp 448-469. Departamento de Geografía, Universidad de Alcalá.

Vicente-Serrano, S. M., Beguería, S., López-Moreno, J. I., García-Vera, M. A. \& Stepanek, P. 2009. A complete daily precipitation database for northeast Spain: reconstruction, quality control, and homogeneity. International Journal of Climatology. Doi:10.1002/joc.1850.

Viviroli, D. \& Weingartner, R. 2004. The hydrological significance of mountains: from regional to global scale. Hydrology and Earth System Sciences, 8:1016-1029.

Widmann, M. \& Schär, C. 1997. A principal component and long-term trend analysis of daily precipitation in Switzerland. International Journal of Climatology, 17:1333-1356.

Yue, S., Pilon, P. \& Cavadias, G. 2002. Power of the Mann-Kendall and Spearman's rho tests for detecting monotonic trends in hydrological series. Journal of Hydrology, 259:254-271.

Zhang, L., Dawes, W. R. \& Walker, G. R. 2001. Response of mean annual evapotranspiration to vegetation changes at catchment scale. Water Resources Research, 37:701-708.

Zhang, X. B., Vincent, L. A., Hogg, W. D. \& Niitsoo, A. 2000. Temperature and precipitation trends in Canada during the 20th century. Atmosphere-Ocean, 38:395-429. 